Research Paper

\title{
Effectiveness and Safety of Chinese Herbal Injections Combined with Fluoropyrimidine and Oxaliplatin-based Chemotherapy for Advanced Colorectal Cancer: A Systematic Review and Meta-analysis of 63 Randomized Controlled Trials
}

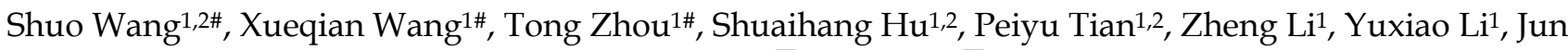 \\ Dong1, Yuerong Gui ${ }^{1}$, Dandan Wang ${ }^{1}$, Ying Zhang ${ }^{\circledR}$, Wei Hou ${ }^{1 凶}$ \\ 1. Department of Oncology, China Academy of Chinese Medical Sciences Guang'anmen Hospital, Beijing, China. \\ 2. Beijing University of Chinese Medicine, Beijing, China. \\ \#Co-first authors with equal contributions to this work. \\ 凹 Corresponding authors: Wei Hou (E-mail: houwei1964@163.com) and Ying Zhang (E-mail: zylzy501@163.com). \\ ( ) The author(s). This is an open access article distributed under the terms of the Creative Commons Attribution License (https://creativecommons.org/licenses/by/4.0/). \\ See http://ivyspring.com/terms for full terms and conditions.
}

Received: 2021.03.26; Accepted: 2021.09.29; Published: 2021.10.25

\begin{abstract}
Purpose: To investigate effectiveness and safety of Chinese herbal injections ( $\mathrm{CHIs}$ ) in conjunction with fluoropyrimidine and oxaliplatin-based chemotherapy (FOBC) for advanced colorectal cancer (CRC).

Methods: A comprehensive search was conducted in 7 electronic databases for related randomized controlled trials (RCTs) from inception to April 30, 2021. The quality of each trial was assessed according to the Cochrane Handbook for Systematic Reviews of Interventions, the differences in effectiveness and safety outcomes between two groups were evaluated, and the results were expressed as the risk ratios (RRs) and 95\% confidence interval $(\mathrm{Cl})$. Subgroup analyses were performed according to the types of $\mathrm{CHls}$, and Review Manager 5 was used to statistically analyze the outcomes.

Results: 63 studies involving $9 \mathrm{CHIs}$ and 4733 patients were included in this review. The meta-analysis results suggested that compared with $\mathrm{FOBC}$ therapy, $\mathrm{CH}$ s plus FOBC therapy showed significant improvements in objective response rate (ORR) $(R R=1.34,95 \% \mathrm{Cl}: 1.27-1.42, P<0.00001)$, disease control rate (DCR) $(R R=1.09,95 \% \mathrm{Cl}$ : 1.06-1.11, $P<0.00001), 1$-year survival rate $(\mathrm{RR}=2.27,95 \% \mathrm{Cl}$ : $1.23-4.18, P=0.009)$ and quality of life ( $Q \circ L)(R R=1.21,95 \% C l: 1.14-1.28, P<0.00001)$, and decreases in the incidence of chemotherapy-induced leukopenia ( $R R=0.64,95 \% \mathrm{Cl}: 0.50-0.82, P<0.0005)$, nausea and vomiting ( $R R=0.65,95 \% \mathrm{Cl}: 0.51-0.83, P=0.0005)$ and diarrhea $(R R=0.34,95 \% \mathrm{Cl}: 0.20-0.58, P<0.0001)$.

Conclusion: From the evidence available, CHls could increase ORR, DCR and 1-year survival rate, improve QoL and relieve chemotherapy-induced leukopenia, nausea and vomiting and diarrhea when combined with FOBC in advanced CRC treatment, Nevertheless, on account of the limitations, more rigorous RCTs with high-quality methodology were needed to further confirm the results.
\end{abstract}

Key words: advanced colorectal cancer, Chinese herbal injections, effectiveness, randomized controlled trial, systematic review

\section{Introduction}

Colorectal cancer (CRC), the third most common cancer worldwide, is a serious threat to people's health and life. CRC is the second leading cause of death among cancers, with an estimated 935,173 deaths, counting $9.4 \%$ of all cancer deaths [1]. Although surgery remains the primary treatment for CRC, $50 \%$ of patients recur or metastasize after radical resection. More than $25 \%$ of the patients confirmed in 
its advanced stage [2], with the overall 5-year survival rate ranges from 10 to $18 \%$. Fluoropyrimidines and oxaliplatin-based chemotherapy (FOBC) is the first-line treatment for patients with advanced CRC, some cases can benefit from FOBC to improve survival as well as locoregional control [3]. However, studies have demonstrated that it often accompanied by adverse reaction, further leading a reduced quality of life.

In Asia, Traditional Chinese Medicine (TCM) is a considerable adjuvant treatment for advanced CRC in combination with chemotherapy, and has been shown to increase effectiveness and reduce side effects. Chinese herbal injection ( $\mathrm{CHI})$, prepared by extracting and purifying effective ingredients from Chinese herbal medicines, is an important part of TCM [4]. It breaks the limitations of the traditional delivery way of Chinese herbal medicines via oral administration, but intravenous injection instead, thus has the advantages of high bioavailability, high blood concentration, rapid action and no digestive tract absorption process. Many researches indicated that it has obvious advantages in improving short-term effectiveness, enhancing life quality and reducing chemotherapy-related toxicity. However, previous studies commonly focused on an individual $\mathrm{CHI}$, while the types of CHIs are various, and the effectiveness and safety of all potential CHIs still remains inconclusive [5]. Thus, a systematic review was designed to fill this knowledge gap by quantitatively synthesizing the evidence. The aim was to evaluate the effectiveness and safety of all potential CHIs for treating advanced CRC and to provide help for clinical medication in the future.

\section{Materials and Methods}

This systematic review and meta-analysis was developed according to the Preferred Reporting Items for Systematic Reviews and Meta-Analysis (PRISMA) guidelines, and has been registered through International Platform of Registered Systematic Review and Meta-analysis Protocols (INPLASY) as INPLASY2020100050. The complete study protocol was previously published [6]. Ethical approval was not required as all the research materials were published studies.

\section{Eligibility criteria}

Only randomized controlled trials (RCTs) were selected and assessed for inclusion based on the following eligibility criteria: (1) Types of Participants: Patients were cytologically or pathologically confirmed cases of CRC and belong to Stage III or IV according to American Joint Committee on Cancer Staging System ( $8^{\text {th }}$ edition) or mentioned "advanced". (2) Types of Interventions: Control groups received FOBC that contained fluoropyrimidine and oxaliplatin, and the fluoropyrimidine drugs include 5-fluorouracil (5-FU) and Capecitabine. Treatment groups received CHIs plus FOBC therapy. In each trial, the FOBC regimen was eligible and the same in both treatment and control group. CHIs were given intravenously. (3) Types of Outcomes: The primary outcomes were objective response rate (ORR) and disease control rate (DCR). According to World Health Organization (WHO) [7] guidelines for solid tumor responses or Response Evaluation Criteria in Solid Tumors (RECIST) [8], the tumor responses were evaluated as complete response (CR), partial response $(\mathrm{PR})$, stable disease (SD) and progressive disease (PD). ORR refers to the proportion of patients with CR plus PR. DCR calculated as the proportion of patients with CR plus PR plus SD. The secondary outcomes were progression-free survival (PFS), survival rate, quality of life (QoL) and safety outcomes. PFS defined as the time from study entry to relapse or death. Survival rate referred to the proportion of participants alive at the beginning of a time interval who survive to the end of the interval [6]. Improvement of QoL was considered when Karnofsky performance scale (KPS) score increased, or decreased no more than 10 scores after treatment.[14]. Safety outcomes covered the incidence of grade 2 or greater leukopenia, diarrhea, and nausea and vomiting, measured by Standard Classification of WHO or National Cancer Institute Common Terminology Criteria for Adverse Events (NCI-CTCAE). The included studies should reported at least one of the above outcomes of interest.

\section{Information Sources}

A comprehensive search was conducted from inception to April 30, 2021 in 7 electronic medical databases, including PubMed, EMBASE, Cochrane, China National Knowledge Infrastructure (CNKI), Wanfang Data, VIP Database for Chinese Technical Periodicals (VIP) and SinoMed.

\section{Search Strategy}

To identify all potential relevant publications, search terms were constructed for two domains: (1) colorectal cancer, (2) CHIs. The terms used for colorectal cancer contained: "Colorectal Neoplasms[MeSH]", "Colonic Neoplasms[MeSH]", "Rectal Neoplasms[MeSH]", "colorectal cancer", "colorectal neoplasm", "colorectal carcinoma", "colorectal tumor", etc. The following terms were used for CHIs: "Chinese herbal injection", "injection of TCM" and certain CHIs such as "Shenqifuzheng", "Kanglaite", "Fufangkushen", "Compound Kushen", "Cinobufacini", "Xiaoaiping", "Elemene", 
"Lentinan", "javanica oil emulsio", "kangai", "Astragalus", "Shenfu", "Shenmai" and multiple synonyms for each term. More specific search strategies were showed on File S1.

\section{Study Selection}

2 independent reviewers (J Dong and YR Gui) screened all the relevant articles on the basis of titles and abstracts. The full texts were scanned for further elimination based on the eligibility criteria. All disagreements were resolved by consensus. All relevant articles were managed in NOTEEXPRESS software.

\section{Data extraction}

2 reviewers ( $\mathrm{T}$ Zhou and $\mathrm{SH} \mathrm{Hu}$ ) completed the data extraction in Excel software independently, and the following items were extracted: general information including author, year, publication, sample size, detailed information of participants, intervention measures and outcomes. The disagreements between the 2 reviewers were settled by S Wang and Y Zhang.

\section{Risk of bias and quality assessment}

We evaluated methodological quality of the included articles according to "Risk of Bias Assessment Tool" of the Cochrane Handbook for Randomized Controlled Trials [9]. The risk of bias was evaluated in 7 items including random sequence generation, allocation concealment, blinding of participants and personnel, incomplete outcome data, selective reporting, and other sources of bias, and finally evaluated as "low risk," "unclear risk," or "high risk" [10]. PY Tian and Z Li assessed the studies' quality independently, any differences were decided by XQ Wang and W Hou. The Grading of Recommendations, Assessment, Development and Evaluation (GRADE) system was used to grade the quality of evidence [11].

\section{Summary measures and data synthesis}

Review Manager 5.3 was used to conduct statistical analyses. Risk ratios (RRs) were used to evaluate effectiveness and safety for dichotomous outcomes with $95 \%$ confidence intervals (CI). P values $<0.05$ were considered to indicate statistical significance. Subgroup analyses were performed according to the types of CHIs and presented with pooled data simultaneously. The heterogeneity was judged based on the $I^{2}$ value and $P$ value. If the studies had non-significant heterogeneity within the studies or subgroups $\left(I^{2}<50 \%, P>0.1\right)$, we used a fixed effects model. If there was great heterogeneity within the studies or subgroups $\left(I^{2}>50 \%, P<0.1\right)$, we used the random effects model. If the data quantitative synthesis was not possible, we analyzed the available data qualitatively.

\section{Risk of Bias across trials}

Funnel-plots were used to assess publication bias when the number of the included trials was more than 10.

\section{Additional analyses}

To determine the robustness of results, sensitivity analyses were conducted based on the quality of trials [12], participants' number, treatment duration of CHIs, stage of cancer and publication year. Trial sequential analysis (TSA) was used to calculate the required information size (RIS) in the meta-analysis.

\section{Results}

\section{Study Selection}

The study selection process was described in Figure 1. A total of 8334 articles were identified from the initial literature search. After removing duplicates and irrelevant articles, 480 articles remained. Through reviewing the full texts of the remaining, a total of 63 papers [13-75] finally reached the criteria for entrance into the meta-analysis.

\section{Study Characteristics}

63 RCTs recruiting 4733 patients were included. The baseline characteristics of the included trials were summarized in Table 1. All trials were conducted in China. All participants enrolled were patients with advanced CRC. There were 2394 and 2339 patients in the experimental and control groups, respectively. The number of participants in each RCT varied from 36 to 250. The numbers of studies included for 9 different CHIs were as follows: Compound Kushen injection (26 trials) [14, 17, 19, 21, 23, 27, 33, 34, 39-41, 43, 46, 47, 48, 51, 54, 55, 59-61, 65, 67, 68, 70, 71]; Aidi injection (15 trials) $[16,18,20,22,26,28,35,37,42,44$, $45,53,56,64,69]$; Shenqifuzheng injection (11 trials) $[24,25,30,31,36,38,52,58,66,73,75]$; Kanglaite injection (3 trials) $[57,62,63]$; Cinobufacini injection (3 trials) $[13,29,50]$; Xiaoaiping injection (2 trials) [32, 72]; Javanica oil emulsion injection (1 trial) [49]; Astragalus injection (1 trial) [15]; Lentinan injection (1 trial) [74]

\section{Quality evaluation}

The results of the methodological evaluation were shown in Figure 2. With regard to random sequence generation, 22 studies $[13,17,19,22,24,26$, $27,32,34,36,37,47,48,50,52,56,60,62,67,69,72,73]$ were assessed as "low risk" because random number table and stratified randomization were adopted, the 
other studies did not report any randomization procedure, and were evaluated as "unclear". Regarding allocation concealment, 1 trial [47] was evaluated as "low risk" because web-based central allocation was adopted, the risk of remaining RCTs were unclear.

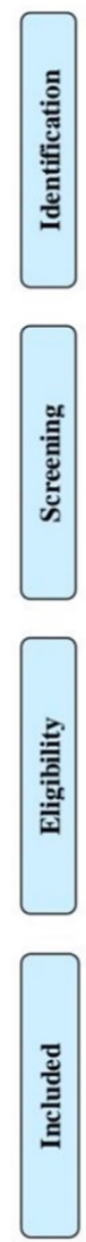

Records identified through database searching $(n=8334)$ : $\operatorname{CNKI}(\mathrm{n}=2215)$; VIP( $n=1667)$; Wanfang

Data( $\mathrm{n}=3515)$; SinoMed( $\mathrm{n}=674)$;

PubMed(n=119); EMBASE(n=104);

Cochrane $(n=40)$

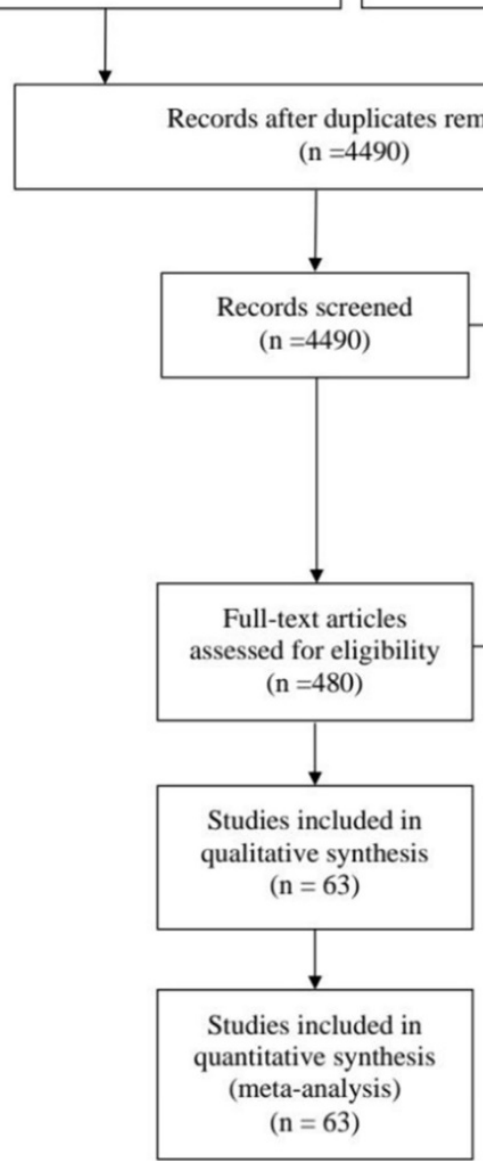

Additional records identified through other sources $(\mathrm{n}=0)$

Records excluded $(\mathrm{n}=4010)$ : Not CHIs $(n=293)$

Not CRC $(n=304)$

Experimental study $(\mathrm{n}=1902)$

Literature reviews $(\mathrm{n}=810)$

Summary of experience $(n=413)$

Meta-analysis $(\mathrm{n}=121)$

Research of classics literature $(\mathrm{n}=167)$

Full-text articles excluded with reasons $(n=417)$ :

Not advanced CRC $(\mathrm{n}=53)$

Not relevant interventions $(\mathrm{n}=76)$

Not relevant controls $(n=14)$

Not relevant outcomes $(\mathrm{n}=22)$ Not RCT $(n=245)$

With error or duplicated data $(n=7)$

Figure 1. Flow diagram of the search for eligible studies.

Table 1. Basic characteristics of the Included Studies

\begin{tabular}{|c|c|c|c|c|c|c|c|c|}
\hline Study ID & $\mathrm{N}(\mathrm{T} / \mathrm{C})$ & Age & $\begin{array}{l}\text { TNM } \\
\text { stages }\end{array}$ & Control group & Intervention group & CHIs dosage & CHIs Treatment & $\begin{array}{l}\text { Interested } \\
\text { outcomes }\end{array}$ \\
\hline Cai HF 2020 & $31 / 31$ & $\begin{array}{l}\text { T: } 70.37 \\
\text { C: } 70.61\end{array}$ & NR & 5-FU+L-OHP+LV & $\mathrm{CI}+5-\mathrm{FU}+\mathrm{L}-\mathrm{OHP}+\mathrm{LV}$ & $20 \mathrm{~mL} /$ day & $\begin{array}{l}21 \text { days/course, } 2-4 \\
\text { courses }\end{array}$ & (1) (2) \\
\hline Cao ZY 2009 & $33 / 29$ & $\begin{array}{l}\text { T: } 53.6 \\
\text { C: } 54.7\end{array}$ & III-IV & 5-FU+L-OHP+LV & CKS+ 5-FU+L-OHP+LV & $15 \mathrm{~mL} /$ day & 28 days/course, 2 courses & (1)(2)(5) (6) \\
\hline Chen F 2009 & $30 / 30$ & $\begin{array}{l}\text { T: } 53.8 \pm 14.4 \\
\text { C: } 52.5 \pm 12.6\end{array}$ & IV & 5-FU+L-OHP+LV & $\mathrm{AS}+5-\mathrm{FU}+\mathrm{L}-\mathrm{OHP}+\mathrm{LV}$ & $60 \mathrm{~mL} /$ day & 21 days/course, 3 courses & (1)(2)(5)(6)(7) \\
\hline Chen LF 2013 & $30 / 30$ & $\begin{array}{l}\text { T: } 53.6 \\
\text { C: } 54.8\end{array}$ & NR & 5-FU+L-OHP+LV & $\mathrm{AD}+5-\mathrm{FU}+\mathrm{L}-\mathrm{OHP}+\mathrm{LV}$ & $80 \mathrm{~mL} /$ day & 10 days/course, 4 courses & (1)(2) (5)(7) \\
\hline Chen XY 2017 & $35 / 35$ & $\begin{array}{l}\mathrm{T}: 49.36 \\
\mathrm{C}: 42.66\end{array}$ & NR & 5-FU+L-OHP+LV & $\mathrm{AD}+5-\mathrm{FU}+\mathrm{L}-\mathrm{OHP}+\mathrm{LV}$ & $50 \mathrm{~mL} /$ day & 21 days/course, 2 courses & (1)(2) 7 \\
\hline Ding X 2010 & $30 / 30$ & $\begin{array}{l}\text { T: } 64.5 \\
\text { C: } 63\end{array}$ & NR & 5-FU+L-OHP+LV & CKS + 5-FU+L-OHP+LV & $20 \mathrm{~mL} /$ day & 14 days/course, 4 courses & (3)(6) 7 \\
\hline Fan S 2010 & $44 / 44$ & 61 & NR & 5-FU+L-OHP+LV & $\mathrm{AD}+5-\mathrm{FU}+\mathrm{L}-\mathrm{OHP}+\mathrm{LV}$ & 100 mL/day & 14 days/course, 4 courses & (1) \\
\hline Fang XG 2012 & $36 / 36$ & $\begin{array}{l}\mathrm{T}: 49.8 \pm 4.1 \\
\mathrm{C}: 48.6 \pm 3.9\end{array}$ & III-IV & 5-FU+L-OHP+LV & CKS + 5-FU+L-OHP+LV & $15 \mathrm{~mL} /$ day & 14 days/course, 2 courses & (1) (2) (5) \\
\hline Fu H 2020 & $52 / 52$ & $\begin{array}{l}\text { T: } 60.39 \\
\text { C: } 60.08\end{array}$ & NR & $\mathrm{CAP}+\mathrm{L}-\mathrm{OHP}$ & $\mathrm{AD}+\mathrm{CAP}+\mathrm{L}-\mathrm{OHP}$ & $\begin{array}{l}50-100 \\
\mathrm{~mL} / \text { day }\end{array}$ & 14 days/course, 2 courses & (1) (2) \\
\hline Gao W 2010 & $38 / 35$ & NR & IV & 5-FU+L-OHP+LV & CKS+ 5-FU+L-OHP+LV & $20 \mathrm{~mL} /$ day & 10 days/course, 4 courses & (1) (2) (5) \\
\hline Guo HM 2019 & $36 / 36$ & $\mathrm{~T}: 53.03$ & NR & 5-FU+L-OHP+LV & SQFZ+5-FU+L-OHP+LV & 250 mL/day & 14 days/course, 4 courses & (1) (2) \\
\hline
\end{tabular}




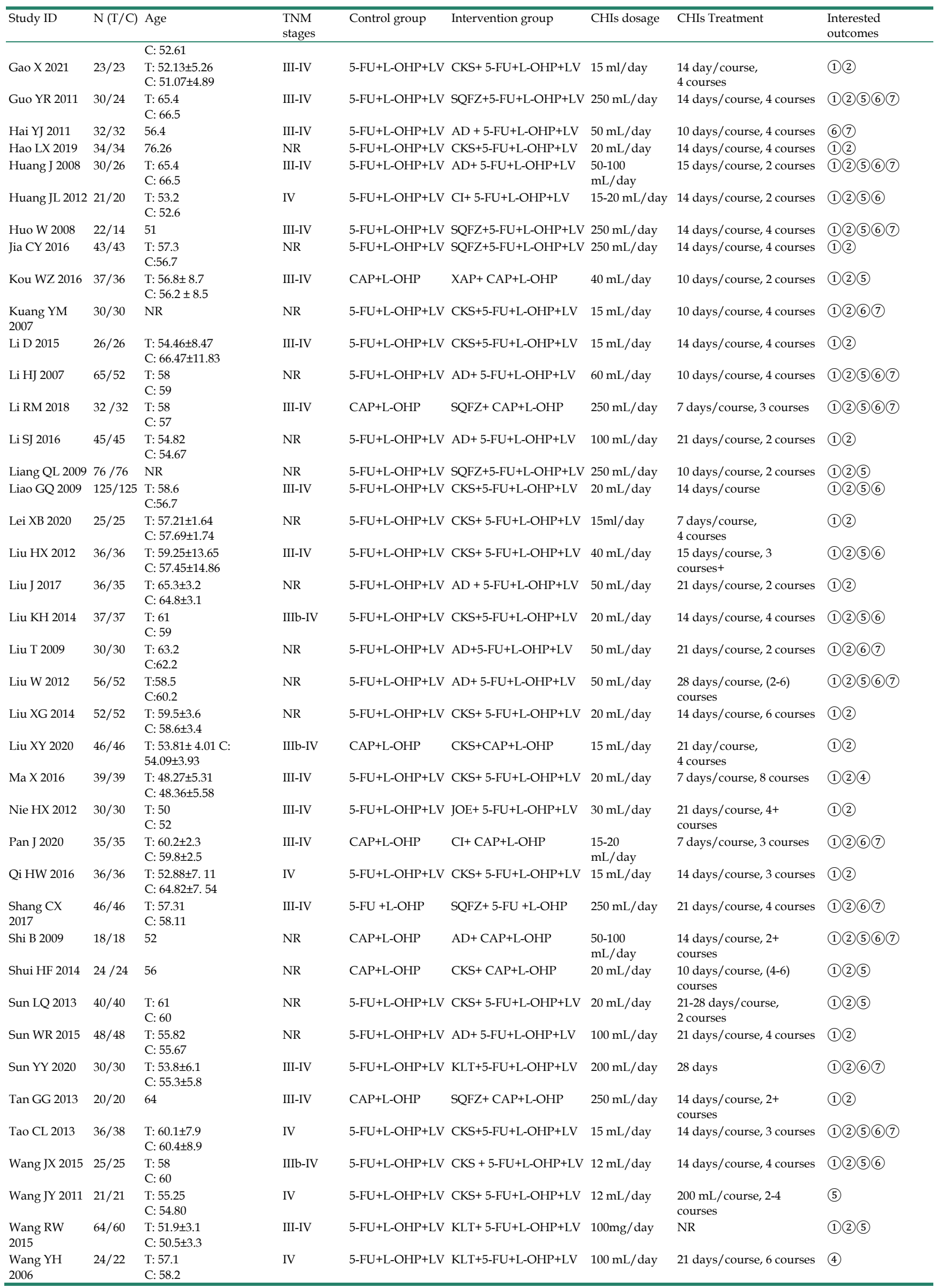




\begin{tabular}{|c|c|c|c|c|c|c|c|c|}
\hline Study ID & $\mathrm{N}(\mathrm{T} / \mathrm{C})$ & Age & $\begin{array}{l}\text { TNM } \\
\text { stages }\end{array}$ & Control group & Intervention group & CHIs dosage & CHIs Treatment & $\begin{array}{l}\text { Interested } \\
\text { outcomes }\end{array}$ \\
\hline Wang YT 2012 & $38 / 36$ & 52 & NR & 5-FU+L-OHP+LV & $\mathrm{AD}+5-\mathrm{FU}+\mathrm{L}-\mathrm{OHP}+\mathrm{LV}$ & $80 \mathrm{~mL} /$ day & 10 days/course, 4 courses & (1) (2) (5) \\
\hline $\begin{array}{l}\text { Weng ML } \\
2020\end{array}$ & $45 / 45$ & $\begin{array}{l}\text { T: } 51.07 \pm 5.21 \\
\text { C: } 50.11 \pm 4.25\end{array}$ & III-IV & 5-FU+L-OHP+LV & $\mathrm{CKS}+5-\mathrm{FU}+\mathrm{L}-\mathrm{OHP}+\mathrm{LV}$ & $20 \mathrm{~mL} /$ day & 7 days/course, 4 courses & (1)(2)(5) (6) \\
\hline Xing F 2015 & $30 / 30$ & $\begin{array}{l}\mathrm{T}: 52 \\
\mathrm{C}: 53\end{array}$ & III-IV & 5-FU+L-OHP+LV & $\mathrm{SQFZ}+5-\mathrm{FU}+\mathrm{L}-\mathrm{OHP}+\mathrm{LV}$ & $250 \mathrm{~mL} /$ day & 10 days/course, 4 courses & (1)(2) (5) \\
\hline Yan Q 2015 & $41 / 41$ & $\begin{array}{l}\text { T: } 55.1 \\
\text { C: } 53.6\end{array}$ & NR & 5-FU+L-OHP+LV & $\mathrm{CKS}+5-\mathrm{FU}+\mathrm{L}-\mathrm{OHP}+\mathrm{LV}$ & $20 \mathrm{~mL} /$ day & 7 days/course, 4 courses & (1)(2) (5) \\
\hline Yang J 2015 & $39 / 39$ & $\begin{array}{l}\text { T: } 55.1 \\
\text { C: } 53.8\end{array}$ & IV & $\mathrm{CAP}+\mathrm{L}-\mathrm{OHP}$ & $\mathrm{CKS}+\mathrm{CAP}+\mathrm{L}-\mathrm{OHP}$ & $12 \mathrm{~mL} /$ day & 7 days/course, 4 courses & (1)(2) (5) \\
\hline Yu ZH 2016 & $43 / 43$ & $\begin{array}{l}\text { T:54.59 } \\
\text { C:4.85 }\end{array}$ & NR & 5-FU+L-OHP+LV & $\mathrm{CKS}+5-\mathrm{FU}+\mathrm{L}-\mathrm{OHP}+\mathrm{LV}$ & $40 \mathrm{~mL} /$ day & 15 days/course, 4 courses & (1)(2) \\
\hline Zan L 2015 & $40 / 40$ & $\begin{array}{l}\mathrm{T}: 52 \\
\mathrm{C}: 51\end{array}$ & III-IV & 5-FU+L-OHP+LV & $\mathrm{CKS}+5-\mathrm{FU}+\mathrm{L}-\mathrm{OHP}+\mathrm{LV}$ & $30 \mathrm{~mL} /$ day & 7 days/course, 2 courses & (1)(2) (5) \\
\hline Zhang L 2012 & $20 / 20$ & $\begin{array}{l}\text { T: } 59.24 \pm 20.37 \\
\text { C: } 61.56 \pm 21.53\end{array}$ & III-IV & 5-FU+L-OHP+LV & $\mathrm{XAP}+5-\mathrm{FU}+\mathrm{L}-\mathrm{OHP}+\mathrm{LV}$ & $1 \mathrm{mg} /$ day & 7 days/course, 2 courses & (1) (2) 5 \\
\hline Zhang Q 2021 & $65 / 65$ & $\begin{array}{l}\text { T: } 59 \pm 10 \\
\text { C: } 59 \pm 10\end{array}$ & III-IV & 5-FU+L-OHP+LV & $\mathrm{AD}+5-\mathrm{FU}+\mathrm{L}-\mathrm{OHP}+\mathrm{LV}$ & $50 \mathrm{ml} /$ day & $\begin{array}{l}14 \text { days/course, } \\
4 \text { courses }\end{array}$ & (1) (2) \\
\hline Zhang W 2015 & $43 / 43$ & 64.3 & III-IV & $\mathrm{CAP}+\mathrm{L}-\mathrm{OHP}$ & SQFZ+ CAP+L-OHP & 250 mL/day & 14 days/course, 2 courses & (1)(2) \\
\hline $\begin{array}{l}\text { Zhang ZH } \\
2011\end{array}$ & $38 / 34$ & $\begin{array}{l}\text { T: } 58 \\
\text { C: } 59\end{array}$ & NR & 5-FU+L-OHP+LV & $\mathrm{LE}+5-\mathrm{FU}+\mathrm{L}-\mathrm{OHP}+\mathrm{LV}$ & $1 \mathrm{mg} /$ day & 14 days/course, 8 courses & (1)(2) (7) \\
\hline Zhao T 2011 & $32 / 32$ & NR & NR & 5-FU+L-OHP+LV & $\mathrm{SQFZ+5-FU+L-OHP+LV}$ & 250 mL/day & 14 days/course, 2 courses & (1)(2)(5)(7) \\
\hline
\end{tabular}

Random sequence generation (selection bias)

Allocation concealment (selection bias)

Blinding of participants and personnel (performance bias)

Blinding of outcome assessment (detection bias)

Incomplete outcome data (attrition bias)

Selective reporting (reporting bias)

Other bias
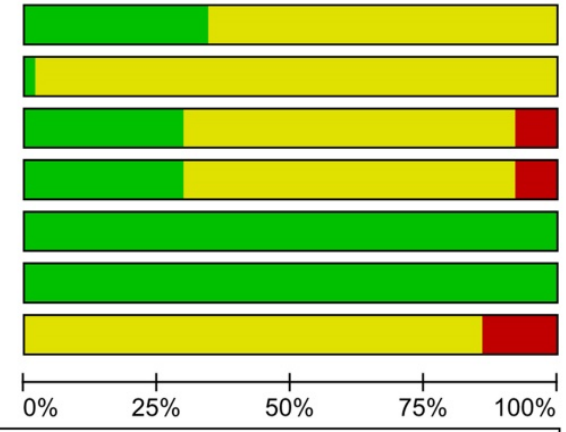

$\square$ Low risk of bias $\quad \square$ Unclear risk of bias $\quad \square$ High risk of bias

Figure 2. Risk of bias graph.

Regarding blinding, since placebos were not mentioned in any of the studies, blinding was considered not performed in any of them. However, when the outcomes were ORR and DCR, it was considered that clinical judgements would not be influenced by lack of blinding because outcomes measured based on imaging. Therefore, 19 studies [17, $20,22,27,31,34,37,40,46,47,48,51,56,58,62,69,70$, 72,73 ] that only reported ORR and DCR with clearly diagnostic criteria were evaluated as "low risk". 40 studies [13-16, 18, 21, 23-25, 28-30, 32, 33, 35, 36, 38, 39, $41-45,49,50,52-55,57,59,61,64-68,71,74,75]$ in which subjective assessments were included in outcomes, making estimation of the influence of blinding on the study results difficult, were evaluated as "unclear". 4 studies [19, 26, 60, 63] that only performed subjective assessments were evaluated as "high risk", since blinding could have affected the study results.
The risk of incomplete outcome data was low as the reported data was consistence with the stated randomized numbers. The risk of selective reporting was low because the outcome results reported just as description in methods. Regarding other bias, 9 trials $[13,39,45,49,53-55,58,62]$ took a range like " $2-6$ " to limit the courses instead a definite figure or did not mention duration, which was a potential source of bias and were assessed as "high risk", the other studies were not clear [76].

The results of the GRADE evaluation of studies which evaluated effectiveness and safety were presented in Table 2. All the reasons for downgrading are labeled [77].

\section{Effectiveness and safety}

The findings of the meta-analyses were summarized in Table 3, and subgroup analyses conducted according to categories of $\mathrm{CHIs}$ were shown in Table 4. 
Table 2. The results of GRADE evaluation

\begin{tabular}{|c|c|c|c|c|c|c|c|c|}
\hline Quality assessment & Numbers of RCTs & Risk of bias & Inconsistency & Indirectness & Imprecision & Publication bias & RR $(95 \% \mathrm{CI})$ & Certainty of evidence \\
\hline ORR & 59 & serious $^{\mathrm{a}}$ & not serious $\left(I^{2}=0 \%, P=0.98\right)$ & not serious & not serious & strongly suspected $^{\mathrm{d}}$ & $1.34(1.27-1.42)$ & LOW \\
\hline DCR & 58 & serious $^{a}$ & not serious $\left(I^{2}=0 \%, P=0.71\right)$ & not serious & not serious & strongly suspected ${ }^{\mathrm{d}}$ & $1.09(1.06-1.11)$ & LOW \\
\hline 1-year survival rate & 2 & serious $^{a}$ & not serious $\left(I^{2}=0 \%, P=0.54\right)$ & not serious & very seriousc & undetected & $2.27(1.23-4.18)$ & VERY LOW \\
\hline Quality of life & 30 & very serious ${ }^{\mathrm{a}}$ & serious $^{\mathrm{b}}\left(I^{2}=32 \%, P=0.05\right)$ & not serious & not serious & strongly suspected $^{\mathrm{d}}$ & $1.21(1.14-1.28)$ & VERY LOW \\
\hline Leukopenia & 20 & seriousa & serious $^{\mathrm{b}}\left(I^{2}=53 \%, P=0.004\right)$ & not serious & not serious & strongly suspected ${ }^{\mathrm{d}}$ & $0.64(0.50-0.82)$ & VERY LOW \\
\hline Nausea and vomiting & 13 & serious $^{a}$ & not serious $\left(I^{2}=0 \%, P=1.00\right)$ & not serious & seriousc & strongly suspected ${ }^{\mathrm{d}}$ & $0.65(0.51-0.83)$ & VERY LOW \\
\hline Diarrhea & 12 & serious ${ }^{a}$ & not serious $\left(I^{2}=0 \%, P=0.94\right)$ & not serious & Not serious & strongly suspected ${ }^{\mathrm{d}}$ & $0.34(0.20-0.58)$ & LOW \\
\hline
\end{tabular}

a Unclear description of the hidden methods of random sequence and random allocation.

b Point estimates vary widely from study to study.

c The number of studies was too small and the confidence interval was too wide to be accurate.

$\mathrm{d}$ The funnel plots were asymmetrical, which indicated that publication bias might influence the results of the analysis.

Table 3. Summary of the Meta-analysis

\begin{tabular}{|c|c|c|c|c|c|c|c|}
\hline \multirow[t]{2}{*}{ Outcomes } & \multirow[t]{2}{*}{ Studies } & \multirow[t]{2}{*}{ Participants } & \multirow[t]{2}{*}{ Statistical methods } & \multirow[t]{2}{*}{ Pooled RRs (95\% CI) } & \multirow[t]{2}{*}{$P$} & \multicolumn{2}{|c|}{ Heterogeneity } \\
\hline & & & & & & $I^{2}$ & $P_{h}$ \\
\hline$\overline{\text { ORR }}$ & 59 & 4595 & FEM & $1.34(1.27-1.42)$ & $<0.00001$ & $0 \%$ & 0.98 \\
\hline DCR & 58 & 4507 & REM & $1.09(1.06-1.11)$ & $<0.00001$ & $0 \%$ & 0.71 \\
\hline 1-year survival rate & 2 & 124 & FEM & $2.27(1.23-4.18)$ & 0.009 & $0 \%$ & 0.54 \\
\hline QoL & 30 & 2217 & REM & $1.21(1.14-1.28)$ & $<0.00001$ & $32 \%$ & 0.05 \\
\hline Leukopenia & 20 & 1504 & REM & $0.64(0.50-0.82)$ & 0.0005 & $53 \%$ & 0.004 \\
\hline Nausea and vomiting & 13 & 955 & FEM & $0.65(0.51-0.83)$ & 0.0005 & $0 \%$ & 1.00 \\
\hline Diarrhea & 12 & 785 & FEM & $0.34(0.20-0.58)$ & $<0.0001$ & $0 \%$ & 0.94 \\
\hline
\end{tabular}

FEM: fixed-effects model; CI: confidence interval; RRs: risk ratios; REM: random-effects model.

Table 4. Subgroup analyses of all outcomes

\begin{tabular}{|c|c|c|c|c|c|c|}
\hline \multirow[t]{2}{*}{ Subgroups } & \multirow[t]{2}{*}{ Number of studies } & \multirow{2}{*}{$\begin{array}{l}\text { Pooled RRs } \\
(95 \% \mathrm{CI})\end{array}$} & \multirow[t]{2}{*}{ Z } & \multirow[t]{2}{*}{$P$} & \multicolumn{2}{|c|}{ Heterogeneity } \\
\hline & & & & & $I^{2}$ & $P_{h}$ \\
\hline \multicolumn{7}{|l|}{ ORR } \\
\hline Compound Kushen injection & 24 & $1.41(1.30,1.54)$ & 7.89 & $<0.00001$ & $0 \%$ & 0.87 \\
\hline Aidi injection & 14 & $1.19(1.07,1.31)$ & 3.38 & 0.0007 & $0 \%$ & 1.00 \\
\hline Shenqifuzheng injection & 11 & $1.38(1.20,1.60)$ & 4.37 & $<0.0001$ & $0 \%$ & 0.94 \\
\hline Kanglaite injection & 2 & $1.61(1.18,2.20)$ & 3.02 & 0.003 & $0 \%$ & 0.94 \\
\hline Cinobufacini injection & 3 & $1.44(1.17,1.78)$ & 3.43 & 0.0006 & $0 \%$ & 0.72 \\
\hline Xiaoaiping injection & 2 & $1.56(0.97,2.53)$ & 1.82 & 0.07 & $0 \%$ & 0.41 \\
\hline \multicolumn{7}{|l|}{ DCR } \\
\hline Compound Kushen injection & 24 & $1.10(1.06,1.13)$ & 5.37 & $<0.00001$ & $0 \%$ & 0.83 \\
\hline Aidi injection & 13 & $1.07(1.02,1.12)$ & 2.99 & 0.003 & $0 \%$ & 0.71 \\
\hline Shenqifuzheng injection & 11 & $1.11(1.02,1.21)$ & 2.43 & 0.01 & $40 \%$ & 0.08 \\
\hline Kanglaite injection & 2 & $1.19(0.93,1.51)$ & 1.36 & 0.17 & $55 \%$ & 0.14 \\
\hline Cinobufacini injection & 3 & $1.08(1.00,1.18)$ & 1.91 & 0.06 & $0 \%$ & 0.59 \\
\hline Xiaoaiping injection & 2 & $1.20(0.99,1.44)$ & 1.89 & 0.06 & $0 \%$ & 0.59 \\
\hline \multicolumn{7}{|l|}{ QoL } \\
\hline Compound Kushen injection & 13 & $1.20(1.08,1.34)$ & 3.48 & 0.0005 & $62 \%$ & 0.002 \\
\hline Aidi injection & 7 & $1.25(1.13,1.38)$ & 4.30 & $<0.0001$ & $0 \%$ & 0.85 \\
\hline Shenqifuzheng injection & 6 & $1.21(1.08,1.35)$ & 3.25 & 0.001 & $0 \%$ & 0.56 \\
\hline Xiaoaiping injection & 2 & $1.16(0.94,1.43)$ & 1.37 & 0.17 & $0 \%$ & 0.36 \\
\hline \multicolumn{7}{|l|}{ Leukopenia } \\
\hline Compound Kushen injection & 5 & $0.66(0.40,1.08)$ & 1.67 & 0.10 & $75 \%$ & 0.003 \\
\hline Aidi injection & 6 & $0.62(0.43,0.89)$ & 2.59 & 0.010 & $0 \%$ & 0.95 \\
\hline Shenqifuzheng injection & 5 & $0.72(0.47,1.12)$ & 1.47 & 0.14 & $0 \%$ & 0.89 \\
\hline \multicolumn{7}{|l|}{ Nausea and vomiting } \\
\hline Compound Kushen injection & 2 & $0.58(0.35,0.96)$ & 2.11 & 0.03 & $0 \%$ & 0.61 \\
\hline Aidi injection & 5 & $0.65(0.45,0.94)$ & 2.27 & 0.02 & $0 \%$ & 0.94 \\
\hline Shenqifuzheng injection & 4 & $0.63(0.35,1.15)$ & 1.50 & 0.13 & $0 \%$ & 0.88 \\
\hline \multicolumn{7}{|l|}{ Diarrhea } \\
\hline Aidi injection & 6 & $0.31(0.16,0.61)$ & 3.41 & 0.0006 & $0 \%$ & 0.86 \\
\hline Shenqifuzheng injection & 3 & $0.24(0.06,0.97)$ & 2.00 & 0.05 & $0 \%$ & 0.72 \\
\hline
\end{tabular}

CI: confidence interval; DCR: disease control rate; ORR: objective response rate; RRs: risk ratios; QoL: quality of life. 


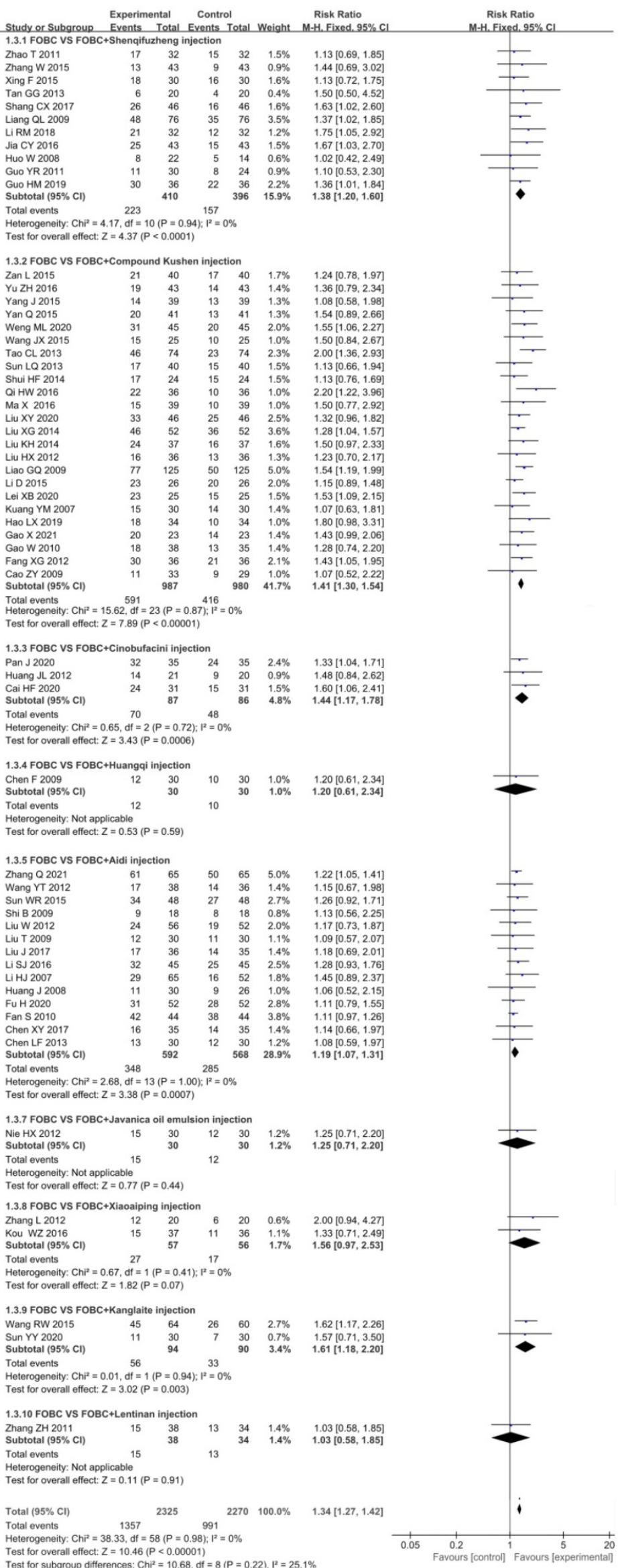

Figure 3. Forest plot of $O R R$ in $F O B C$ versus FOBC plus $C H$ ls. 


\section{Objective response rate (ORR)}

Data from 59 RCTs [13-18, 20-25, 27-48, 49-59, 61, 62, 64-75] with 9 types of CHIs contributed to the evidence for ORR. No statistical significant heterogeneity $\left(I^{2}=0 \%, P=0.98\right)$ was found and a fixed effect model was adopted. The results showed that the ORR was significantly enhanced in CHIs plus FOBC group when compared with $\mathrm{FOBC}$ group. ( $\mathrm{RR}=1.34$, 95\%CI: 1.27-1.42, $P<0.00001$ ) (Figure 3). Subgroup analysis stratified by types of CHIs showed that the ORR was significantly enhanced in Compound Kushen injection subgroup (RR=1.41, 95\% CI: 1.30-1.54, $\left.P<0.00001 ; I^{2}=0 \%\right)$, Aidi injection subgroup (RR=1.19, 95\% CI: 1.07-1.31， $\left.P=0.0007 ; \quad I^{2}=0 \%\right)$, Shenqifuzheng injection subgroup ( $R R=1.38,95 \% \mathrm{CI}$ : 1.20-1.60, $\left.P<0.0001 ; I^{2}=0 \%\right)$, Cinobufacini injection subgroup $(\mathrm{RR}=1.44,95 \% \mathrm{CI}: 1.17-1.78, \quad P=0.0006$; $I^{2}=0 \%$, and Kanglaite injection subgroup $(R R=1.61$, 95\% CI: 1.18-2.20, $P=0.003 ; I^{2}=0 \%$, while showed no advantage in Xiaoaiping injection subgroup $(R R=1.56$, 95\% CI: 0.97-2.53, $P=0.07 ; I^{2}=0 \%$ ).

\section{Disease control rate (DCR)}

In total, 58 RCTs [13-18, 21-25, 27-48, 49-59, 61, 62, 64-75] with 9 CHIs contributed to the analysis of DCR with no significant heterogeneity $\left(I^{2}=0 \%\right.$, $P=0.71)$. The results showed that the DCR was significantly enhanced in CHIs plus FOBC group than that in FOBC group. (RR=1.09, 95\%CI: 1.06-1.11, $P$ $<0.00001$ ) (Figure 4). Subgroup analysis indicated that DCR was enhanced in Compound Kushen injection subgroup (RR=1.10, 95\% CI:1.06-1.13, P<0.00001; $\left.I^{2}=0 \%\right)$, Shenqifuzheng injection subgroup $(R R=1.11$, 95\% CI: 1.02-1.21, $\left.P=0.01 ; I^{2}=40 \%\right)$, Aidi injection subgroup $\quad(R R=1.07,95 \%$ CI: 1.02-1.12, $P=0.003$; $I^{2}=0 \%$, while showed no advantage in Cinobufacini injection subgroup $(\mathrm{RR}=1.08,95 \% \mathrm{CI}$ : 1.00-1.18, $\left.P=0.06 ; \quad I^{2}=0 \%\right), \quad$ Kanglaite injection subgroup (RR=1.19, 95\% CI:0.93-1.51， $\left.P=0.17 ; \quad I^{2}=55 \%\right)$ and Xiaoaiping injection subgroup $(R R=1.20,95 \% C I$ : $0.99-1.44, P=0.06 ; I^{2}=0 \%$ ).

\section{Survival rate and Progression-free Survival (PFS)}

There were 2 trials [47, 63] reported 1-year survival rate. The results indicated that the 1-year survival rate in $\mathrm{CHIs}$ plus $\mathrm{FOBC}$ group was higher than that in FOBC group ( $R R=2.27,95 \% \mathrm{CI}: 1.23-4.18$, $P=0.009)$ with low heterogeneity $\left(I^{2}=0 \%, P=0.54\right)$ (Figure 5).

2 RCTs [19, 69] reported progression-free survival (PFS), while because of the unextractable data and/or the diversity of survival outcomes in the included RCTs, meta-analysis was not possible for it
[78].

\section{Quality of life (QoL)}

The data on the QoL was available for 30 trials $[14,16,21,23,25,26,28-30,32,35,36,38,39,41,43,45$, $49,53-55,60,61,64,66-68,71,72,75]$ involving 6 types of CHIs. The results showed that the QoL in CHIs plus FOBC group was significantly higher than that in FOBC group (RR=1.21, 95\% CI: 1.14-1.28, $P<0.00001)$ with low heterogeneity $\left(I^{2}=32 \%, P=0.05\right)$. Subgroup analysis indicated that QoL was significantly improved in Compound Kushen injection subgroup (RR=1.20, 95\% CI: 1.08-1.34, $\left.P=0.0005 ; I^{2}=62 \%\right)$, Aidi injection subgroup $(\mathrm{RR}=1.25,95 \% \mathrm{CI}: 1.13-1.38$, $\left.P<0.0001 ; \quad I^{2}=0 \%\right)$ and Shenqifuzheng injection subgroup (RR=1.21, 95\% CI: 1.08-1.35, $P=0.001$; $I^{2}=0 \%$, while showed no advantage in Xiaoaiping injection subgroup ( $R R=1.16,95 \%$ CI: 0.94-1.43, $P=0.17 ; I^{2}=0 \%$ ) (Figure 6).

\section{Leukopenia}

19 studies $[15,19,25,26,28-30,33,35,36,39$, $43-45,49,52,53,59,75]$ with 6 types of CHIs reported the incidence of chemotherapy-induced leukopenia. Figure 7 showed that the incidence of leukopenia in CHIs combined with FOBC group was lower than that in FOBC group ( $R R=0.64,95 \%$ CI: $0.50-0.82$, $P=0.0005)$ with obvious heterogeneity $\left(I^{2}=53 \%\right.$, $P=0.004)$. Subgroup analysis showed that the incidence of leukopenia was decreased in Aidi injection subgroup $(\mathrm{RR}=0.62,95 \% \mathrm{CI}$ : $0.43-0.89, P=0.01$; $I^{2}=0 \%$ ), but showed no advantage in Compound Kushen injection subgroup (RR=0.66, 95\% CI: $\left.0.40-1.08, \quad P=0.10 ; \quad I^{2}=75 \%\right) \quad$ and Shenqifuzheng injection subgroup $(\mathrm{RR}=0.72,95 \% \mathrm{CI}$ : 0.47-1.12, $P=0.14 ; I^{2}=0 \%$ ).

\section{Nausea and Vomiting}

A total of 13 studies [15, 19, 25, 26, 28, 30, 35, 36, $45,49,52,53,59]$ with 5 types of CHIs reported the data of nausea and vomiting, Figure 8 exhibited that the incidence of nausea and vomiting in CHIs plus FOBC group was lower than that in FOBC alone group (RR=0.65, 95\% CI: 0.51-0.83, $P=0.0005$. heterogeneity: $\left.I^{2}=0 \%, P=1.00\right)$. Subgroup analysis showed that the incidence of nausea and vomiting was decreased in Aidi injection subgroup $(R R=0.65$, 95\% CI: $\left.0.45-0.94, P=0.02 ; I^{2}=0 \%\right)$ and Compound Kushen injection subgroup ( $R=0.58, \quad 95 \%$ CI: $0.35-0.96, P=0.03 ; I^{2}=0 \%$ ), but showed no advantage in Shenqifuzheng injection subgroup $(\mathrm{RR}=0.63,95 \% \mathrm{CI}$ : $\left.0.35-1.15, P=0.13 ; I^{2}=0 \%\right)$. 


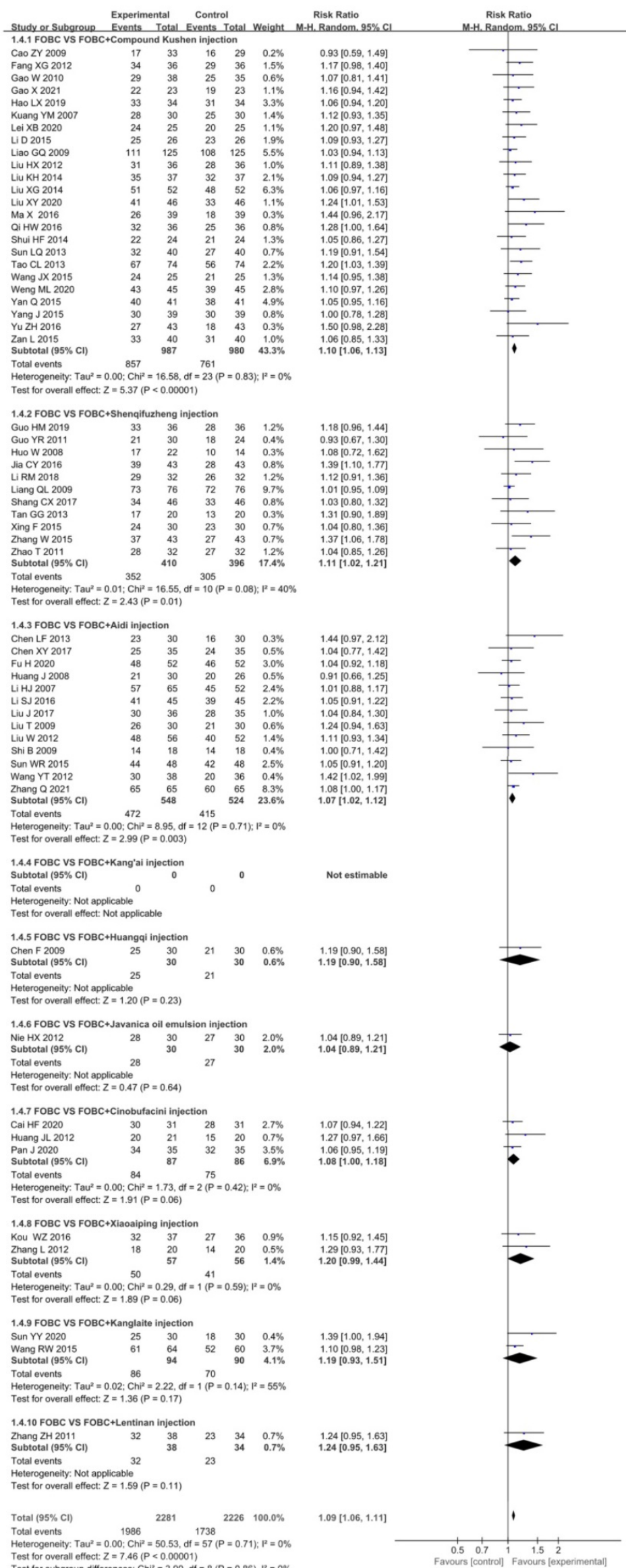

Figure 4. Forest plot of DCR in FOBC versus FOBC plus $\mathrm{CHls}$. 


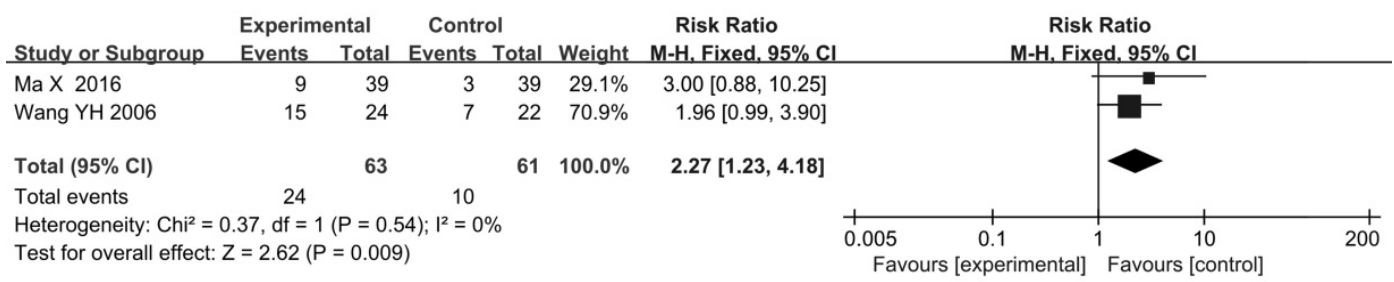

Figure 5. Forest plot of 1-year survival rate in $\mathrm{FOBC}$ versus $\mathrm{FOBC}$ plus $\mathrm{CH}$ ls.

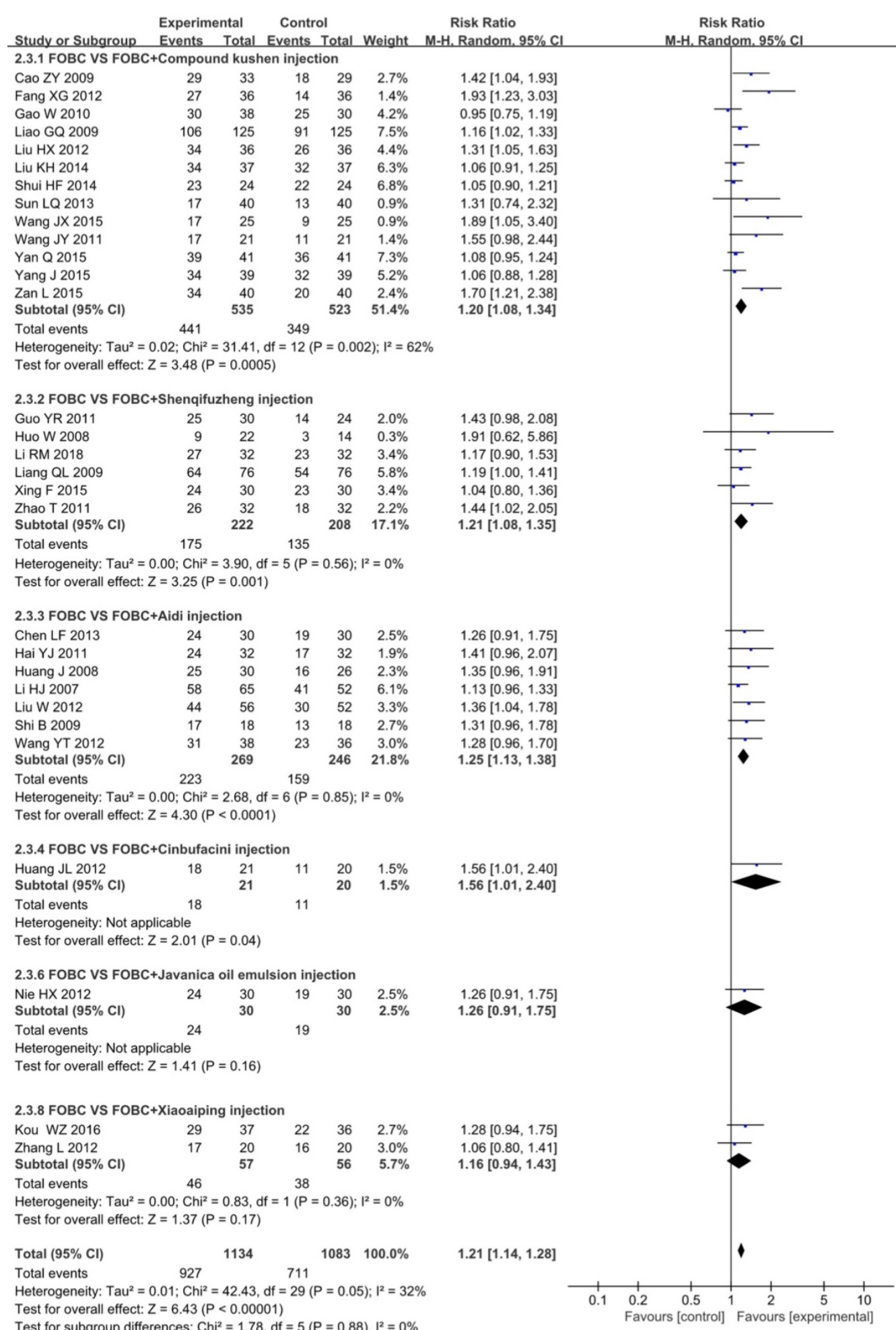

Figure 6. Forest plot of $\mathrm{QoL}$ in FOBC versus FOBC plus $\mathrm{CH}$ ls. 


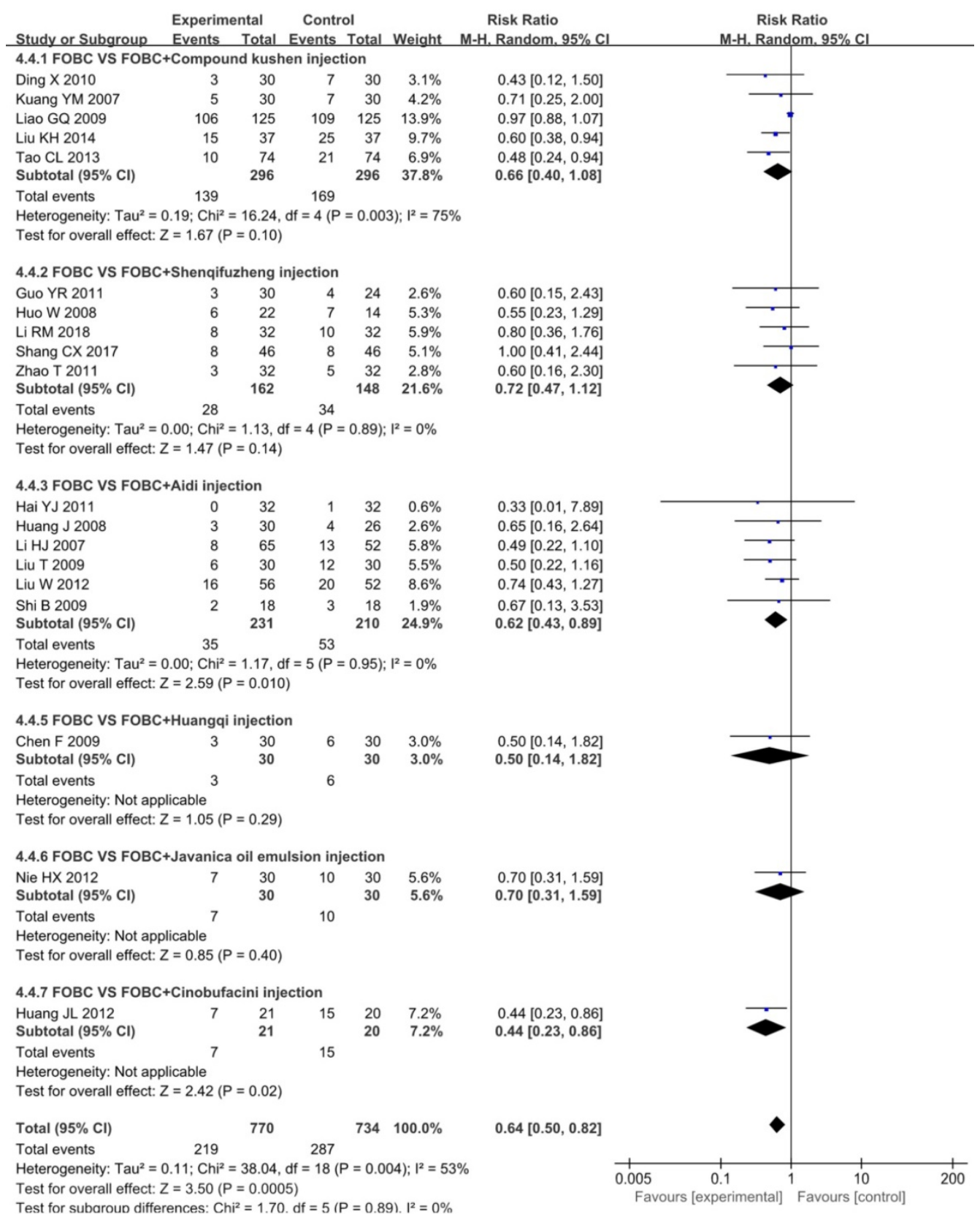

Figure 7. Forest plot of leukopenia in $F O B C$ versus $F O B C$ plus $C H$ ls.

\section{Diarrhea}

A total of 12 RCTs $[15,18,25,26,28,30,33,35,36$, $45,49,53]$ with 5 types of CHIs reported diarrhea, Figure 9 showed that the incidence of diarrhea in CHIs plus FOBC group was lower than that in FOBC alone group $(\mathrm{RR}=0.34,95 \% \mathrm{CI}: 0.20-0.58, P<0.0001$. heterogeneity: $\left.I^{2}=0 \%, P=0.94\right)$. In subgroup analysis, the incidence of diarrhea was decreased in Shenqifuzheng injection subgroup $(\mathrm{RR}=0.24,95 \% \mathrm{CI}$ : 0.06-0.97, $P=0.05 ; I^{2}=0 \%$ ) and Aidi injection subgroup (RR=0.31, 95\% CI: 0.16-0.61, $P=0.0006 ; I^{2}=0 \%$ ).

\section{Publication bias}

Figure 10A-F showed the funnel plots based on the data of the ORR, DCR, QoL, leukopenia, nausea and vomiting and diarrhea were asymmetrical, which indicated that publication bias might influence the results of the analysis.

\section{Additional analyses}

Regarding ORR, the primary outcome, the pooled data showed that CHIs plus FOBC increased ORR significantly (RR=1.34, 95\%CI: 1.27-1.42, $P<0.00001)$. Similar increases were observed when the sensitivity analyses were performed based on the 
results of Cochrane Risk of Bias Tool (excluded 9 RCTs [13, 39, 45, 49, 53-55, 58, 62] of poor quality with at least one "high risk" domain in risk of bias assessment) (RR=1.33, 95\% CI: 1.26-1.42, $P<0.00001)$, participants number (only included 8 RCTs with $\geq 50$ participants in each group) ( $R R=1.35,95 \% \mathrm{CI}$ : $1.22-1.49, P<0.00001)$, treatment duration of CHIs (only included $30 \mathrm{RCTs}$ with $\geq 4$ courses) ( $R R=1.30$, 95\% CI: 1.21-1.40, P<0.00001), publication year (only included 21 studies published within 5 years) ( $R R=1.40,95 \%$ CI: $1.29-1.53, \mathrm{P}<0.00001$ ) or stage (only included 6 studies enrolled patients with stage IV) ( $R R=1.59,95 \%$ CI: 1.28-1.97, $\mathrm{P}<0.0001)$. It showed that the results of the primary outcome were robust before and after removing related trials (Table 5).
Table 5. Sensitivity analysis of objective response rate (ORR)

\begin{tabular}{|c|c|c|c|c|c|c|}
\hline Types & $\begin{array}{l}\text { Excluded trials } \\
\text { (references) }\end{array}$ & $\begin{array}{l}\text { Remaining } \\
\text { trials }\end{array}$ & $\begin{array}{l}\text { Statistical } \\
\text { methods }\end{array}$ & $\begin{array}{l}\text { RRs } \\
(95 \% \mathrm{CI})\end{array}$ & $P$ & $I^{2}$ \\
\hline $\begin{array}{l}\text { Quality of } \\
\text { trials }\end{array}$ & $\begin{array}{l}9[13,39,45,49, \\
53-55,58,62]\end{array}$ & 50 & FEM & $\begin{array}{l}1.33(1.26, \\
1.42)\end{array}$ & $\begin{array}{l}< \\
0.00001\end{array}$ & $0 \%$ \\
\hline $\begin{array}{l}\text { Participants } \\
\text { number }\end{array}$ & $\begin{array}{l}51[13-18,20,21, \\
23-25,27-34,36,37, \\
40-44,47-59,61, \\
64-68,70-75]\end{array}$ & 8 & FEM & $\begin{array}{l}1.35(1.22 \\
1.49)\end{array}$ & $\begin{array}{l}< \\
0.00001\end{array}$ & $0 \%$ \\
\hline $\begin{array}{l}\text { Treatment } \\
\text { duration of } \\
\text { CHIs }\end{array}$ & $\begin{array}{l}29[13-15,18,21,22, \\
28,29,32,36-39,41, \\
42,44,45,50,51,53, \\
55,57-59,62,71-73, \\
75]\end{array}$ & 30 & FEM & $\begin{array}{l}1.30(1.21, \\
1.40)\end{array}$ & $\begin{array}{l}< \\
0.00001\end{array}$ & $0 \%$ \\
\hline $\begin{array}{l}\text { Publication } \\
\text { year }\end{array}$ & $\begin{array}{l}38[14-16,20,21,23, \\
25,28-30,33-35,38, \\
39,41,43-46,49, \\
53-56,58,59,61,62, \\
64,66-68,71-75]\end{array}$ & 21 & FEM & $\begin{array}{l}1.40(1.29 \\
1.53)\end{array}$ & $\begin{array}{l}< \\
0.00001\end{array}$ & $0 \%$ \\
\hline Stage & $\begin{array}{l}53[13,14,16-18, \\
20-22,24,25,27,28, \\
30-50,52-58,61,62, \\
64-67,69-75]\end{array}$ & 6 & FEM & $\begin{array}{l}1.59(1.28 \\
1.97)\end{array}$ & $\begin{array}{l}< \\
0.0001\end{array}$ & $9 \%$ \\
\hline
\end{tabular}

FEM: fixed-effects model; RRs: risk ratios; $\mathrm{CI}$ : confidence interval.

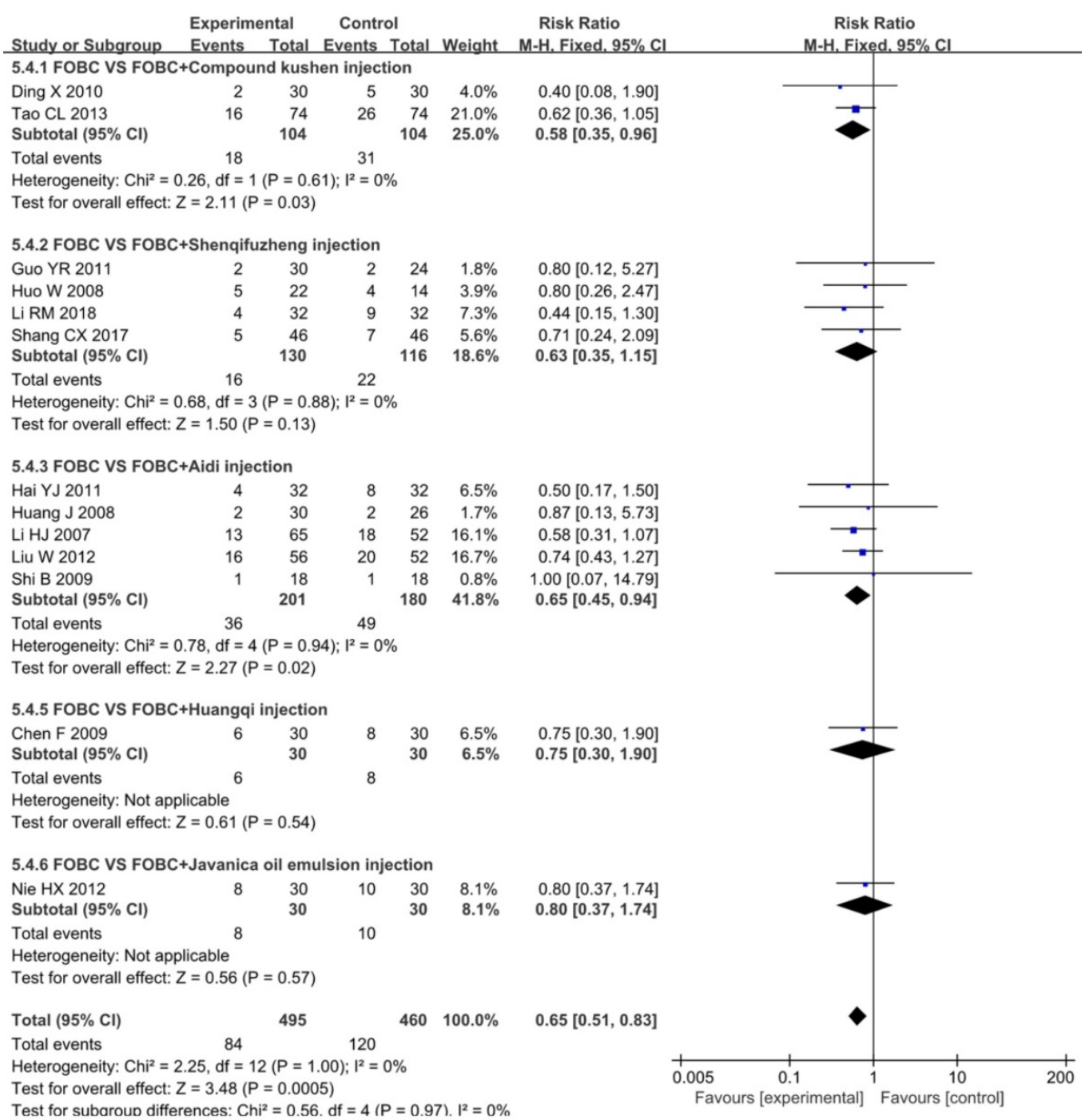

Figure 8. Forest plot of nausea and vomiting in $\mathrm{FOBC}$ versus $\mathrm{FOBC}$ plus $\mathrm{CH}$ ls. 


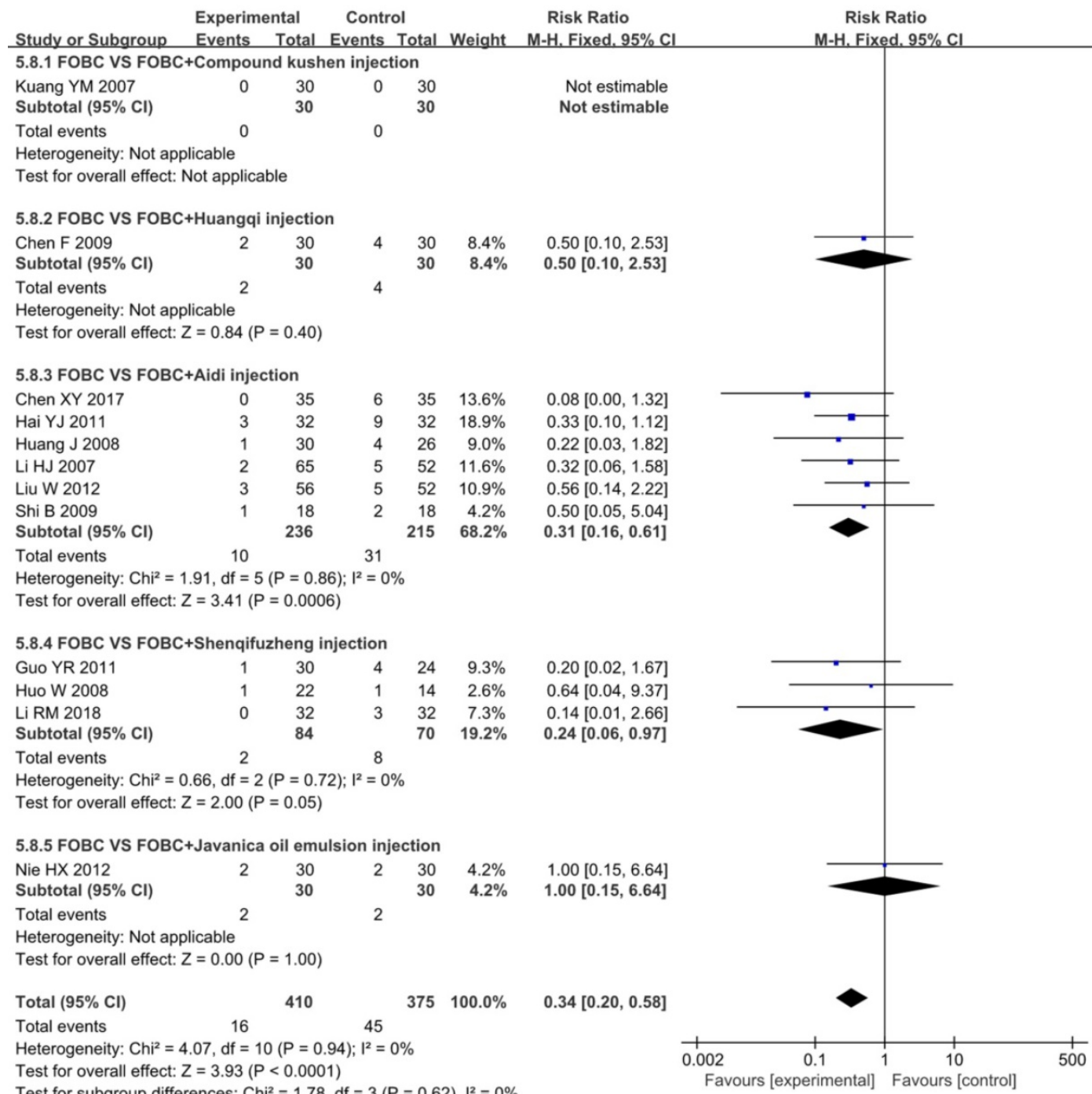

Figure 9. Forest plot of diarrhea in $\mathrm{FOBC}$ versus $\mathrm{FOBC}$ plus $\mathrm{CH}$ ls.
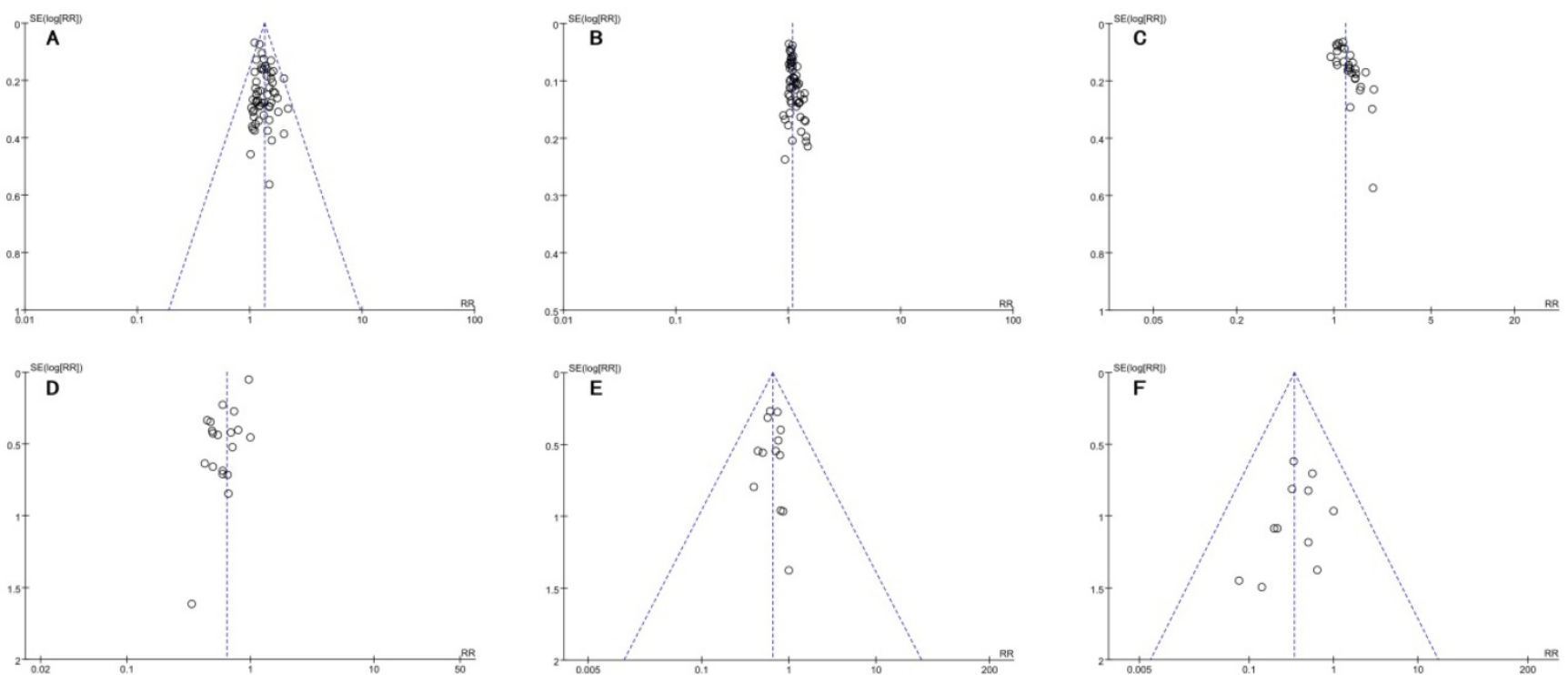

Figure 10. Funnel plots of outcomes: A. ORR; B. DCR; C. QoL; D. leukopenia; E. nausea and vomiting; F. diarrhea. 


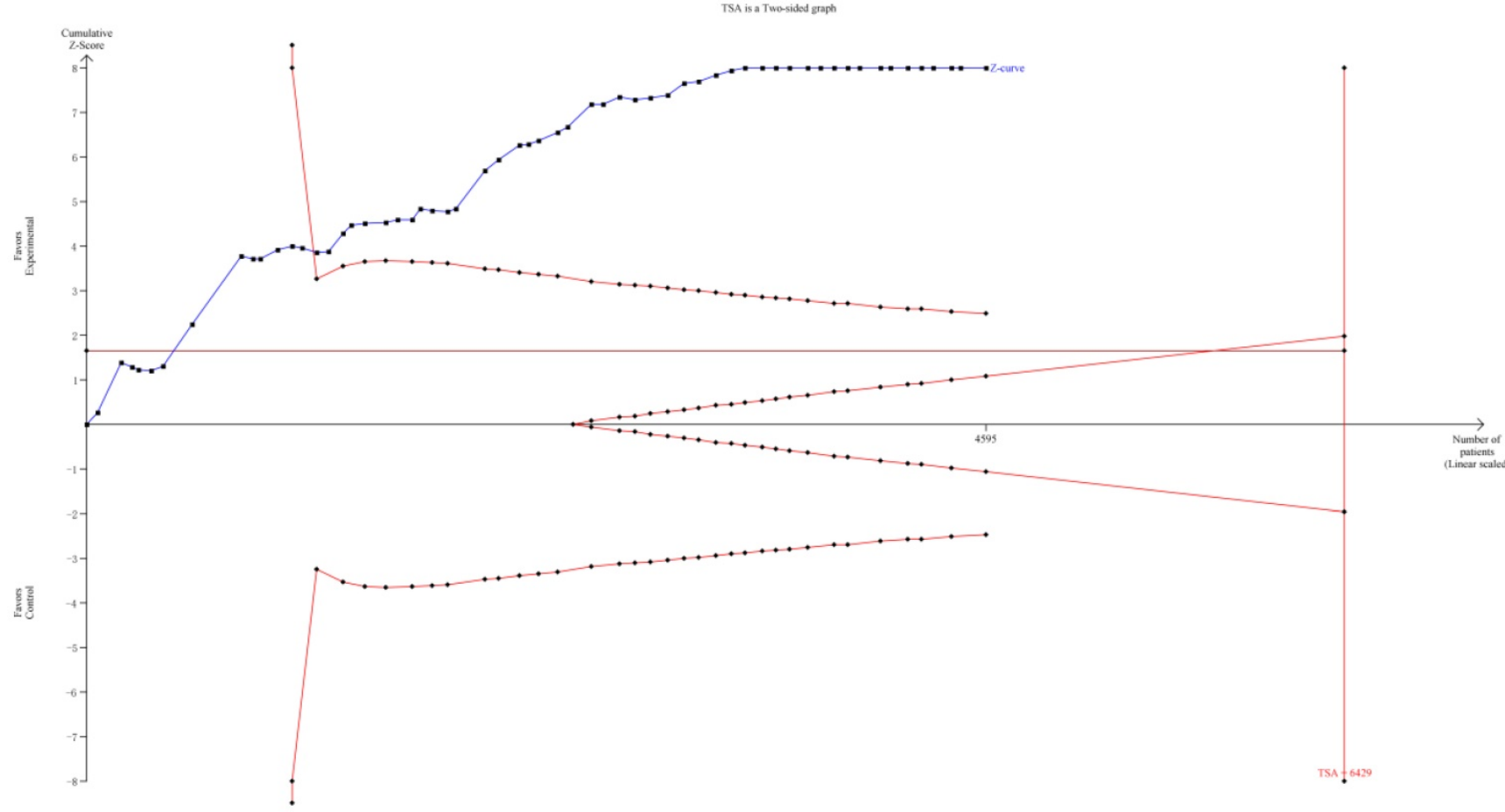

Figure 11. Trial sequential analysis (TSA) on ORR.

TSA was implemented to evaluate the required information size (RIS). As it showed in Figure 11, although the RIS has not been reached, the positive conclusion was obtained in advance as Z-curve had crossed the traditional boundary and TSA boundary. Therefore, it could be thought that $\mathrm{CHIs}$ combined with FOBC was significantly superior to FOBC alone in improving ORR, and the evidence was reliable [79].

\section{Discussion}

To explore the effectiveness and safety of CHIs combined with FOBC in advanced CRC treatment, we conducted this meta-analysis to analyze the evidence in published RCTs. In general, the results of our study indicated that $\mathrm{CHIs}$ in conjunction with $\mathrm{FOBC}$ showed significant improvements in ORR, DCR, 1-year survival rate and QoL; and decreases in incidence of leukopenia, diarrhea and nausea and vomiting, while because of the unextractable data, whether PFS was improved remained unknown. In terms of methodology, the overall quality of the included studies could be considered moderate. GRADE assessments showed a low-quality of evidence. Most results showed low heterogeneity and good robustness.

Although FOBC regimen has been shown to prolong survival and reduce the advent of major complications in patients with advanced CRC [3], due to the lack of anti-tumor selective effects, it also has damaging effects on normal cells while suppressing tumor growth. Thus enhancing therapeutic effects and reducing adverse reactions became an urgent problem in current advanced CRC treatment [80], and CHIs proven to have those effects according to our results. As the products of the combination of TCM and modern science and technology, CHIs not only retain the characteristics of Chinese medicines under the guidance of Chinese medicine theory, but also obtain the advantages of modern chemical medicine like stable composition and fast onset. Compound Kushen injection is extracted from Kushen (Radix sophorae flavescentis) and Baituling (Rhizoma smilacis glabrae), it is extensively used for the treatment of malignant tumor such as liver cancer, lung cancer, and gastrointestinal cancer, and has been found to has the potiential to induce tumor cell differentiation and apoptosis and to inhibit tumor angiogenesis [81]. The main active compounds of Aidi injection include cantharidin, astragalosides, ginsenosides, isofraxidin and syringin that are derived from Chinese herbs, various studies have shown that Aidi injection in relation to anti-tumor activity, immune regulatory action and adverse events relieving [82]. Shenqifuzheng injection is composed of extracts from astragalus membranaceus and codonopsis pilosula, and was clinically indicated to improve body immunity and suppress tumor growth [83]. Regarding effectiveness and safety of $\mathrm{CHIs}$, our review indicated that some CHIs showed great beneficial impact on enhancing short-term effectiveness, improving 1-year survival rate and QoL, and reliving adverse effects. 
To identify certain effective CHIs, we conducted subgroup analyses for all outcomes according to different types of $\mathrm{CHIs}$ as predefined. For the primary outcomes - ORR and DCR, 3 CHIs showed great advantages including Compound Kushen injection, Shenqifuzheng injection and Aidi injection. We also concerned that Xiaoaiping injection that extracted from Marsdenia tenacissima revealed no advantage in ORR, DCR, QoL and adverse reaction improvement, which suggested it might not recommended in the treatment of advanced CRC considering insufficient evidence.

There were some limitations in this study. First, all trials were conducted in China, which might lead to an unavoidable regional bias. Second, publication bias might exist on account of the asymmetrical funnel plots. Third, some studies lacked methodological details in randomization, allocation concealment and blinding, which might result in the emergence of bias and overestimation of effectiveness [84]. Last, the study periods were generally short, and majority of the included trials did not report long-term endpoint outcomes such as overall survival (OS) and PFS that played a vital role in judging the therapeutic effects among patients with tumors. Given the limited quality and quantity of the included studies, more rigorous RCTs with high-quality methodology and long-term endpoint outcomes were needed to verify the beneficial role of CHIs combined with first-line chemotherapy in patients with advanced CRC.

\section{Conclusion}

In conclusion, from the available evidence, $\mathrm{CHIs}$ could increase ORR and DCR, improve 1-year survival rate and QoL, and relieve leukopenia, nausea and vomiting and diarrhea when combined with FOBC in advanced CRC treatment. Meanwhile, considering the limitations, clinicians should choose carefully when applying the conclusions of this study.

\section{Abbreviations}

CHIs: Chinese herbal injections; CI: Confidence Interval; CNKI: China National Knowledge Infrastructure; CR: complete response rates; CRC: colorectal cancer; DCR: disease control rate; FOBC: fluoropyrimidines and oxaliplatin-based chemotherapy; FUs: Fluoropyrimidines; GRADE: Grading of Recommendations, Assessment, Development and Evaluation; IARC: International Agency for Research on Cancer; INPLASY: International Platform of Registered Systematic Review and Meta-analysis Protocols; KPS: Karnofsky performance scale; NCI-CTCAE: National Cancer Institute Common Terminology Criteria for Adverse Events; NIH:
National Cancer Institute; RR: risk ratio; ORR: objective response rate; OS: overall survival; PD: progressive disease; PR: partial response; PFS: progression-free survival; PRISMA: Preferred Reporting Items for Systematic Reviews and Meta-Analysis; QoL: quality of life; RCT: randomized controlled trial; RECIST: Response Evaluation Criteria in Solid Tumors; SD: stable disease; TCM: Traditional Chinese Medicine; TSA: Trial sequential analysis; WHO: World Health Organization; 5-FU: 5-fluorouracil.

\section{Supplementary Material}

Supplementary search strategies. https://www.jcancer.org/v12p7237s1.pdf

\section{Acknowledgements}

This project was funded by Oncology Project of Building Evidence Based Practice Capacity for TCM (No. 60101), National Natural Science Foundation of China (No. 82004179) and The Fundamental Research Funds for the Central Public Welfare Research Institutes (No. 2020YJSZX-3). The sponsors were not involved in design, execution, or writing the study.

\section{Author contributions}

- Shuo Wang, Xueqian Wang, Tong Zhou: collecting data, statistical analysis, writing the article, final approval of the article;

- Tong Zhou, Shuaihang Hu: data extraction, statistical analysis;

- Peiyu Tian, Zheng Li: quality evaluation, final approval of the article;

- Yuxiao Li, Dandan Wang: developing search strategy, critical revision;

- Jun Dong, Yuerong Gui: developing criteria, study selection;

- Wei Hou, Ying Zhang: design, supervising the whole process, critical revision of the article, final approval of the article.

\section{Competing Interests}

The authors have declared that no competing interest exists.

\section{References}

1. [Internet]. International Agency for Research on Cancer. Latest global cancer data: Cancer burden rises to 19.3 million new cases and 10.0 million cancer deaths in 2020. https://www.iarc.who.int/faq/latest-global-cancer-data2020-qa/

2. Zhu R, Yao Y, Gong Y. Advances in TCM research on recurrence and metastasis of colorectal cancer. Hunan J Tradit Chin Med. 2009; 36:191-194.

3. Sterpetti AV, Costi U, D'Ermo G. National statistics about resection of the primary tumor in asymptomatic patients with Stage IV colorectal cancer and unresectable metastases. Need for improvement in data collection. A systematic review with meta-analysis. J Surg Oncol. 2020; 33:11-18.

4. Dong JT. Zhang BL. The use of traditional Chinese medicine injection for critically ill patients can help to turn the tide. Clin J Chin Med. 2020; 06:7. 
5. Zhang D, Ni M, Wu J, et al. The Optimal Chinese Herbal Injections for Use With Radiotherapy to Treat Esophageal Cancer: A Systematic Review and Bayesian Network Meta-Analysis. Front Pharmacol. 2019; 9:1470.

6. Wang S, Wang X, Zhang Y, et al. Efficacy of Chinese herbal injections combined with fluoropyrimidine and oxaliplatin-based chemotherapy for advanced colorectal cancer: A protocol for systematic review and metaanalysis of randomized controlled trials. Medicine. 2020; 99: e23550.

7. Miller AB, Hoogstraten B, Staquet M, et al. A. Reporting results of cancer treatment. Cancer. 1981; 47: 207-214.

8. Therasse P, Arbuck SG, Eisenhauer E. A, et al. New guidelines to evaluate the response to treatment in solid tumors. European Organization for Research and Treatment of Cancer, National Cancer Institute of the United States, National Cancer Institute of Canada. J Natl Cancer Inst. 2000; 92: 205-216.

9. [Internet] Higgins J. http:/ / www.cochrane-handbook.org.

10. Kwon C, Lee, Kim K, et al. Herbal medicine on cancer-related fatigue of lung cancer survivors: Protocol for a systematic review. Medicine. 2020; 99: e18968.

11. Chen S, Bao Y, Xu J, et al. Efficacy and safety of TCM combined with chemotherapy for SCLC: a systematic review and meta-analysis. J Cancer Res Clin Oncol. 2020; 146: 2913-2935.

12. Xiao $Z$, Jiang $Y$, Wang $C Q$, et al. Clinical efficacy and safety of Aidi injection combination with vinorelbine and cisplatin for advanced non-small-cell lung carcinoma: A systematic review and meta-analysis of 54 randomized controlled trials. Pharmacol Res. 2020; 153: 104637.

13. Cai HF, Qiu S. Effect of Cinobufacin injection combined with chemotherapy on serum tumor markers and immune function in elderly patients with colorectal cancer. Chin J Postgrad Med. 2020; 43: 368-372.

14. Cao Z, Pu Y, He J, et al. Clinical Efficacy of Compound Matrine Injection in Combination with FOLFOX-4 Chemotherapy for Advanced Colorectal Cancer. Evaluation and analysis of drug-use in hospitals of China. 2009; 9: 940-941.

15. Chen F. Effect observation of Huangqi injection combined with chemotherapy on quality of life of postoperation patients with advanced stage colorectal carcinoma. Hebei J TCM. 2009; 31: 1696-1698.

16. Chen L. Efficacy of Aidi injection in chemotherapy for advanced colorectal cancer. Chinese J Mod Drug App. 2013; 7: 166-167.

17. Lei $X B$. Effects of Compound Sophora flavestifolia Injection on Immune Function and Serum IL-2, TNF- $\alpha$, INF- $\gamma$ in Advanced Colon Cancer Patients. Modern Medicine and Health Research. 2020; 4: 79-80.

18. Chen X. Analyze the clinical effect of Aidi injection and chemotherapy in the treatment of advanced rectal cancer. Chinese Journal of Ethnomedicine and Ethnopharmacy. 2017; 26: 98-99.

19. Ding X, Xiao XY, Yang XY, et al. Clinical observation of compound Kushen injection combined with FOLFOX4 regime for patients with advanced colorectal cancer. China Oncol. 2010; 20: 860-863.

20. Fan S, Peng L. Clinical observation of Aidi injection combined with FOLFOX-4 regimen in the treatment of advanced colorectal cancer. China Mod Med. 2010; 17:76.

21. Fang XG, Zhang KS. Analysis on the clinic effects of Compound Kushen injection plus chemotherapy on terminal colorectal cancer. Anti-Tumor Pharm. 2012; 2: 293-295.

22. Fu H, Zhang $\mathrm{H}$. Clinical effect of Aidi injection combined with XELOX chemotherapy in the treatment of advanced colorectal cancer. Henan Med Res. 2012; 29: 99-100.

23. Gao W, Li H, Dang Q. Observation on the curative effect of compound Kushen injection combined with chemotherapy in the treatment of metastatic colorectal cancer. Shandong Med J. 2010; 50: 85-86.

24. Guo HM, Zhao ZF. Effect of Shenqifuzheng Injection Combined with FOLFOX4 Chemotherapy on Clinical Efficacy and Serum Related Markers in Patients with Advanced Colon Cancer. Chin J Hemorh 2019; 29: 300-302.

25. Guo Y, Jia J, Zhao H, et al. Shenqifuzheng injection combined with oxaliplatin combined with 5-FU and calcium folate in the treatment of 30 cases of middle and advanced colorectal cancer. Chin Rem Clin. 2010; 11: 221-223.

26. Hai YJ, Ju BL, Du P, et al. Effects of Aidi combined with chemotherapy on immune function and quality of life of advanced colorectal cancer patients. China Pharm. 2011; 14: 1634-1636.

27. Hao L, Hu W, Guo W. Effects of Compound Kushen injection combined with FOLFOX4 chemotherapy regimen on immune function and serum tumor markers in elderly patients with colorectal cancer metastasis. Chinese Journal of Practical Medicine. 2019; 46: 83-85.

28. Huang J, Shen HM, Wang YS. Aidi injection combined with L-OHP + 5-Fu/LV was used to treat 30 cases of middle and advanced colorectal cancer. Henan Tradit Chin Med. 2008; 28: 65-66.

29. Huang J, Lin X. Clinical observation of huachansu injection combined with chemotherapy in the treatment of recurrent metastatic colorectal cancer. Clin Med. 2012; 32: 56-57.

30. Hou W, Li Z, Pan X, et al. Effect of Shenqi Fuzheng injection combined with chemotherapy on advanced colorectal cancer. Chin J Clin Oncol Rehabil.2008; 15: 454-456.

31. Jia C, Chang S, Ma L, et al. Clinical effect and impact on the levels of Treg, TNF- $\alpha$ and IL-12 of Shenqi Fuzheng injection combined with FOLFOX4 for advanced colorectal cancer. Labeled Immunoassays \& Clin Med.2016; 23: 382-384

32. Kou W, Yang X, Zhu X, et al. Effect of Xiaoaiping injection combined with CapeOX regimen for patients with advanced colorectal. Xinxiang Med Univ. 2016; 33: 500-503.
33. Kuang $\mathrm{YM}$, Chen $\mathrm{XB}$, Luo $\mathrm{SX}$, et al. Compound Kushen injection combined with chemotherapy for advanced colorectal cancer. Clin Med. 2007; 27: 36-37.

34. Li D, Zhang Q, Wu S, et al. Clinical study of Compound Kushen injection combined with chemotherapy for advanced colon cancer. Liaoning J Tradit Chin Med. 2015; 42: 1021-1022.

35. Li H, Dong L, Fu S, et al. Clinical observation of Aidi injection combined with FOLFOX 4 chemotherapy in the treatment of advanced colorectal cancer. Chin J Integr Tradit West Med. 2007; 27: 1086-1089.

36. Li R, Shen J. Clinical study of Shenqifuzheng injection combined with CapeOX chemotherapy regimen in treatment of advanced rectal cancer. China Medical Engineering. 2018; 26: 27-31.

37. Li SJ, Chen GF, Fu XF. Clinical observation of Aidi injection combined with FOLFOX4 chemotherapy in the treatment of colon cancer. Jilin Med J. 2016; 37:2023-2024.

38. Liang Q, Pan D, Xie J, et al. Effect of Shenqifuzheng injection combined with chemotherapy in treating advanced colorectal carcinoma. Chin J Integr Tradit West Med. 2009; 29: 439-441.

39. Liao G, Qi Y, Liu P, et al. Compound Matrine Injection in combination with FOLFOX -4 regimen for advanced colorectal cancer: a clinical study. Evaluation and Analysis of Drug-Use in Hospitals of China. 2009; 9: 207-208.

40. Liu XY. Clinical effect of compound sophora flavescera injection combined with XELOX in the treatment of advanced colon cancer. Chronic Pathematology Journal. 2020; 21: 1416-1418.

41. Liu H, Hua X. Treating 36 cases of advanced colorectal cancer with Compound Sophora Sophora injection combined with chemotherapy. Zhejiang Univ Tradit Chin Med. 2012; 47: 225.

42. Liu J, Xu J. Effect of chemotherapy combined with Aidi injection in elderly patients with colorectal cancer. Drugs and Clinic. 2017; 14: 59-61.

43. Liu K, Wang Y, Su H. Observation on the curative effect of compound sophora flavescera injection combined with hemotherapy on advanced colorectal cancer. Henan Journal of Surgery. 2014; 20: 39-40.

44. Liu T, Wang T. Clinical observation of Aidi injection combined with chemotherapy in the treatment of advanced colorectal cancer. Chin J Misdiagn. 2009; 9: 2849-2850.

45. Liu W, Zhong $X$, Wang $K$, et al. Observation of curative effect on Aidi Injection in combination with FOLFOX4 regimen chemotherapy in the treatment of patients with advanced colorectal cancer. China Med Her. 2012; 9: 80-82.

46. Liu XG. Feasibility analysis of compound sophora flavescera injection combined with chemotherapy in the treatment of advanced colon cancer. Chin J Mod Dru Appl. 2014; 8: 150-151.

47. Ma X. Effect analysis of compound Kushen injection and chemotherapy on advanced colorectal cancer. The Med Foru. 2016; 20: 3965-3966.

48. Gao X, Sun HQ, Guo YG, et al. Clinical study of compound sophora flavescera injection combined with chemotherapy for advanced colon cancer. Liaoning J Trad Chin Med. 2021; 48:128-130.

49. Nie HX. Observation on the curative effect of brucea javanica oil emulsion injection combined with FOLFOX4 in the treatment of advanced colon cancer. Chinese Community Doctors. 2012; 14: 56-58.

50. Pan J. Efficacy of huachansu injection combined with chemotherapy in patients with advanced colorectal cancer. Practical Clinical Journal of Integrated Traditional Chinese and Western Medicine. 2020; 20:109-110.

51. Qi HW. Clinical study of Compound Matrine injection combined with chemotherapy in the treatment of patients with advanced colon cancer. Liaoning J Tradit Chin Med. 2016; 43: 547-548.

52. Shang CX, Ma JC, Nan Z, et al. Efficacy of Shenqi Fuzheng injection combined with oxaliplatin in advanced colon cancer and its effect on serum TNF-a and IL-2 levels. Liaoning J Tradit Chin Med. 2017; 44: 1673-1675.

53. Shi $\mathrm{B}, \mathrm{Xu} \mathrm{J}$, Zhang X. Aidi injection combined with XELOX regimen for treatment of advanced colorectal cancer Clinical observation. Mod J Integr Tradit Chin West Med. 2009; 18:2549-2550.

54. Shui HF. Clinical observation on the treatment of liver metastasis of colorectal cancer by Compound Kushen injection combined with XELOX regimen. Medical Information. 2014; 27: 55.

55. Sun LQ. Clinical study of compound sophora flavescens injection combined with chemotherapy in the treatment of metastatic colorectal cancer. Jilin Journal of Chinese Medicine. 2013; 33: 53-54

56. Sun WR, Men ZJ, Guo SL. Clinical study of Aidi injection combined with FOLFOX4 regimen in the treatment of colon cancer. Pharmacol Clin Chin Mater Med. 2015: 31: 245-247.

57. Sun YY, Liang Y, Ye GD. clinical vaule of kanglaite injection combined with FOLFOX-4 regime in patients with advanced colorectal cancer. Med J West China. 2020; 32:1184-1188.

58. Tan GG, Liu L, Li J, et al. Effects of Shenqifuzheng injection on immunologic function of patients with colon cancer, J Hainan Med Coll. 2013;19:627-629.

59. Tao $\mathrm{CL}, \mathrm{Xu}$ JF. Clinical observation on 74 cases of advanced colon cancer treated with compound Kushen injection combined with chemotherapy. Guiding Journal of Traditional Chinese Medicine and Pharmacy. 2013; 19: $42-44$.

60. Wang JY, Cui XM, Du YY, et al. Clinical observation of Compound Kushen injection combined with chemotherapy for advanced colorectal cancer. Chinese J Modern Drug Appl. 2011; 5: 146-147.

61. Wang JX, Xu JC, Xu XH. Observation on treatment of 25 cases of advanced colorectal cancer with Compound Kushen injection combined with chemotherapy. Zhejiang Univ Tradit Chin Med. 2015; 50: 230. 
62. Wang RW. Efficacy and safety analysis of Kanglaite in the treatment of advanced rectal cancer. Medical Information. 2015; 28: 146-147.

63. Wang YH, Li ZY, Gong XQ. Kanglaite injection combined with chemotherapy in the treatment of advanced colorectal cancer. Tumor. 2006; 26:1137-1138.

64. Wang YT, Peng L. Clinical study of Aidi injection combined with FOLFOX4 regimen in the treatment of advanced colorectal cancer. Qingdao Medical Journal. 2012; 44: 173-174.

65. Weng ML, Zheng QH, Chen JX. Effect of Compound Kushen injection combined with oxaliplatin on short-term efficacy and cellular immune function of advanced colon cancer patients. J Zhejiang Univ Tradit Chin Med. 2020; 55: 207-208.

66. Xing F, Feng GA. Effect of Shenqi Fuzheng injection combined with FOLFOX4 in the treatment of advanced colorectal cancer. China Higher Medical Education. 2015; 1: 140-144.

67. Yan Q, Liu QH, Yin YF. Observation on the curative effect of Compound Kushen injection combined with oxaliplatin on rectal cancer. Mod J Integr Tradit Chin West Med. 2015; 24: 3488-3489.

68. Yang J, Han LL, Deng WY, et al. Clinical observation of Compound Kushen injection combined with chemotherapy in the treatment of advanced colorectal carcinoma. Chin J Pract Med. 2015; 42: 76-78.

69. Zhang Q, Ma XB, Li Y. Effect of Aidi injection on clinical efficacy and immune function of patients with middle and advanced colon cancer. Chin Med. 2021;16: 583-587.

70. Yu ZH. Clinical observation of Compound Kushen injection combined with FOLFOX4 in the treatment of advanced colorectal cancer. Chin J Mod Drug Appl. 2016; 10: 173-174.

71. Zan L, Zheng D. Effect analysis of Compound Kushen injection combined with chemotherapy on 40 cases of colorectal cancer. J Clin Psychosom Dis. 2015; $21: 231$.

72. Zhang L. 40 cases of advanced colon cancer treated with Xiaoaiping injection combined with FOLFOX4 regimen. TCM Res. 2012; 25: 14-16.

73. Zhang W, Sun HY, Zheng WE. Effect of Shenqifuzheng injection on quality of life and immune function of advanced colon cancer Patients. Modern Practical Medicine. 2015; 27: 358-359.

74. Zhang $\mathrm{ZH}, \mathrm{Ni} \mathrm{BQ}$, Chen $\mathrm{RX}$. The effect of lentinan injection combined with FOLFOX chemotherapy in treatment of advanced colon cancer. Chinese and Foreign Medical Research 2011; 9: 11-13.

75. Zhao T, Liu Y. Clinical effect of Shenqifuzheng injection combined with mFOLFOX6 in the treatment of advanced colon cancer. Chinese J Modern Drug Appl. 2011; 5: 16-17.

76. Kim KI, Jun JH, Baek $\mathrm{H}$, et al. Oral administration of herbal medicines for radiation pneumonitis in lung cancer patients: A systematic review and meta-analysis. PLoS One. 2018; 13: e0198015.

77. Chen ST, Bao Y, Xu J, et al. Efficacy and safety of TCM combined with chemotherapy for SCLC: a systematic review and meta-analysis. J Cancer Res Clin Oncol. 2020; 146: 2913-2935.

78. Chen H, Yao X, Li T, et al. Compound Kushen injection combined with platinum-based chemotherapy for stage III/IV non-small cell lung cancer: A meta-analysis of 37 RCTs following the PRISMA guidelines. J Cancer. 2020; 11: 1883-1898.

79. Chen $\mathrm{G}, \mathrm{Hu} \mathrm{C}$, Song $\mathrm{Y}$, et al. Relationship between Aurora-A V57I polymorphism and the risk of cancer: a meta-analysis and trial sequential analysis. J Cancer. 2020; 11: 3225-3234.

80. Cao LH, Cai SH, Xu J, et al. Evidence-based pharmaceutical evaluation of the clinical antitumor efficacy of four commonly used traditional Chinese medicine injections. China Pharm. 2014; 25: 268-272.

81. Yu L, Zhou Y, Yang Y, et al. Efficacy and safety of Compound Kushen injection on patients with advanced colon cancer: a meta-analysis of randomized controlled trials. Evid Based Complement Alternat Med. 2017; 2017: 7102514

82. Wang J, Li G, Yu L, et al. Aidi injection plus platinum-based chemotherapy for stage IIIB/IV non-small cell lung cancer: a meta-analysis of 42 RCTs following the PRISMA guidelines. Journal of Ethnopharmacology. 2018; 221:137-150.

83. Jia J, Zhan D, Li J, et al. The contrary functions of IncRNA HOTAIR/ miR-17-5p/PTEN axis and Shenqifuzheng injection on chemosensitivity of gastric cancer cells. Journal of Cellular and Molecular Medicine. 2019; 23: 656-669.

84. Pascual MC, Martinez VV, Álvarez BC, et al. Effectiveness of pharmacological treatments in Duchenne muscular dystrophy: a protocol for a systematic review and meta-analysis. BMJ open. 2019; 9: e029341. 